\title{
Microanalytical characterization of decorations in handmade ancient floor tiles using inductively coupled plasma optical emission spectrometry (ICP-OES)
}

\author{
Santino Orecchio \\ Dipartimento di Chimica “Stanislao Cannizzaro”, Università degli Studi di Palermo, Viale delle Scienze Ed. 17-90128 Palermo, Italy
}

\section{A R T I C L E I N F O}

\section{Article history:}

Received 1 October 2012

Accepted 19 October 2012

Available online 26 October 2012

\section{Keywords:}

Tiles

Ceramics

Pigments

ICP-OES

\begin{abstract}
A B S T R A C T
In this study a total of 114 glazed decorations of 42 ceramic floor tiles, manufactured in Sicily from the 16 th to the 21th AD, were investigated. The micro sampling method, proposed by us, using a cotton swab soaked in hydrofluoric acid, includes advantages of high sensitivity, high selectivity, simplicity, speed, not expensive and can be considered non-destructive because the point of sampling remains invisible to the human eye. ICP-OES technique was used in this study. Twenty-four elements ( $\mathrm{Al}, \mathrm{As}, \mathrm{B}, \mathrm{Be}, \mathrm{Ba}, \mathrm{Ca}, \mathrm{Cd}, \mathrm{Co}, \mathrm{Cr}, \mathrm{Cu}, \mathrm{Fe}, \mathrm{K}$, $\mathrm{Mg}, \mathrm{Mn}, \mathrm{Mo}, \mathrm{Ni}, \mathrm{Pb}, \mathrm{Sb}, \mathrm{Se}, \mathrm{Si}, \mathrm{Sn}, \mathrm{Ti}, \mathrm{V}$ and $\mathrm{Zn}$ ) were determined in each colored enamel.

Enameled decorations in Sicilian tiles were varied in tones and chromatic effects, obtained with a limited number compounds of cobalt, copper, iron, manganese, tin, and in the artifacts following the year 1920, also titanium. An important remark is that some of the colors, such as black, orange, purple and brown were prepared using the same metals, while white, light blue, blue and green were obtained with different elements. The adoption of different recipes for the same color suggests the presence of several laboratories of ceramists working in Sicily who had preferences for different methods for the production of their enameled artifacts.

Concerning the opacifier, the high amounts of lead and antimony, only in a case, indicate the use of lead antimonate $\left(\mathrm{Pb}_{2} \mathrm{Sb}_{2} \mathrm{O}_{7}\right)$. Black decorations were present in several of the analyzed samples and in most of them were identified mixtures of black iron and manganese oxides. The green decorations appear to have been prepared with copper oxide; lighter hue samples contained additional amounts of zinc and/ or barium compounds. Considering all the samples, meanly, iron predominates in yellow decorations, only a sample contains large amounts of $\mathrm{Cd}, \mathrm{Zn}$ and very little $\mathrm{Sb}$, which suggests the use of the pigments $\mathrm{ZnO}$ (as white) and CdS as cadmium yellow. For all samples, except one, we can exclude the use of pigments containing chromium.
\end{abstract}

(c) 2012 Elsevier B.V. All rights reserved.

\section{Introduction}

The application of analytical techniques to the study of historical and cultural heritage has led to a significant progress in the knowledge of human civilizations [1-4]. They also allow us to distinguish between objects which may have a similar visual appearance, but which were made using different raw materials or technological procedures [5-7].

Production of ceramic objects began in Sicily during prehistory and nonstop through the Roman and Medieval periods and still is active today [8]. Reasons for this well-known tile production in Sicily are associated with the presence of widespread utilizable clay deposits and, all at once, to the high level of expertise of local ceramists which has developed over the centuries [8]. The most important manufacturing areas, located in the central-eastern part of Sicily (Gela, Syracuse and Caltagirone), were strongly influenced by decorative motifs of Arabian

\footnotetext{
E-mail address: santino.orecchio@unipa.it.
}

inspiration [8]. Starting from the 16th century, the production of Sicilian ceramic was expanded with the laboratories of Palermo, Sciacca, Burgio, Collesano and Naso. At the end of the 18th century, the pottery production of western Sicily decreased and, in the same period, a new production took place at Santo Stefano di Camastra, on the Tyrrhenian coast of eastern Sicily [8].

The chronological/spatial positioning of a given tile is generally definite by an expert art historian, whose evaluation is based on the style of decorations and on the association of colors and decorative motifs. Only in recent times, a systematic scientific study (archeometry) of Sicilian pottery has been carried out, aiming at a rational classification of local productions, namely understanding of manufacturing techniques and characterizing the composition of the raw materials $[7,8]$.

The decorated ceramic floor tiles are a typical handicraft production. The feature of this kind of artifact is a whitish opaque or shiny glassy coating, usually obtained with white compounds (calcium antimonate, tin dioxide, lead carbonate, etc.) as a substrate for the pigments. Moreover, these objects are usually characterized by contrasting intense and bridge colors. Colors, generally, were obtained 
through metal oxides or synthesized pigments, for example Naples Yellow $\left(\mathrm{Pb}_{3}\left(\mathrm{SbO}_{3}\right)_{2}\right)$, which were used singly or mixed with other pigments. In some cases, the compounds which make the surface opaque, also impart color to the background, for example, copper or copper (I) oxide in red opaque enamel or lead antimonite in yellow ones. The most common pigments present in ancient tiles contain metal ions, such as $\mathrm{Cu}(\mathrm{II}), \mathrm{Fe}(\mathrm{II}), \mathrm{Fe}(\mathrm{III}), \mathrm{Mn}(\mathrm{III}), \mathrm{Mn}(\mathrm{IV})$ and $\mathrm{Co}(\mathrm{II})$ dissolved in the enamel matrix.

The investigation of ancient ceramic technology, which was usually based on the analysis of the ceramic body [7] in the past, can be complemented through the analysis of the pigments used for surface decoration. Despite the importance of the craft ceramics, relatively few scientific studies concerning its pigments have been published $[4,8,9]$. The pigments are among the most interesting matrices to be investigated, because ancient people used many kinds of substances naturally or artificially. The metal identification in the pigments used in ancient works of art is important from the archeometry $[10,11]$ and art history points of view, as well as for the restoration and reproduction of the artifacts.

The problem of pigment identification in ceramics could be resolved by the use of analytical techniques [9-12] some of them require big amounts of samples. The requirement of archeologists and curators for the use of non- or low destructive analytical techniques, during the examination of ancient material, makes inductively coupled plasma optical emission spectrometry (ICP-OES) technique an important tool for the analysis of the cultural heritage. Inductively coupled plasma emission spectrometry is one of the most important multi-elemental quantitative analytical techniques for the study of samples in environmental and cultural heritage sciences $[7,13,14]$.

The present work is aimed to the investigation of ancient and recent ceramic floor tiles, coming from the Sicilian area (Southern Italy), in order to get information about the pigments used to produce the decorations, as a first step to understand the production technology of the Sicilian tile manufacturers. The identification of pigments used in tiles and potteries is important for their reproduction, restoration, dating, and authentication, as well as for obtaining artistic, technical and economic information.

In the present work, we report and discuss the experimental results obtained through ICP-OES measurements performed on a set of forty-two shards of ceramic tiles. In particular, in our case, the sampling procedure involved gently contacting a cotton swab soaked in HF with the decorated enameled surface to chemically remove an infinitesimal quantity of material that does not cause any visible effect to the object.

\section{Experimental}

\subsection{Laboratory equipment}

All glassware and sample containers were thoroughly washed with $\mathrm{HNO}_{3} 2 \%$ solution followed by rinsing with purified water. These were finally kept in the oven at $110{ }^{\circ} \mathrm{C}$ over night. To avoid contaminations of samples, different glassware and pipettes were used for standards and for solutions obtained from samples.

\subsection{Samples}

A set of 42 fragments of glazed ceramic floor tiles (Table 1), manufactured in Sicily in different times from the 16th to the 21th $\mathrm{AD}$ was studied. The choice of samples comprised several of the color motifs available among the great diversity of decorations found in ceramic floor tiles in different periods and production workshops. Fig. 1 shows some samples where the representative colors are depicted. Table 1 includes a full list of the analyzed samples, with the colors analyzed.
Table 1

List of samples analyzed and the identified colors.

\begin{tabular}{|c|c|}
\hline Sample & Identified colors \\
\hline 1a & White-yellow-light blue-green-brown \\
\hline $1 b$ & Green-yellow-brown-light blue \\
\hline $1 \mathrm{bb}$ & Brown \\
\hline $2 a$ & Black-white-blue \\
\hline $5 a$ & Yellow-brown \\
\hline $7 a$ & Green-black \\
\hline $8 \mathrm{a}$ & White-green-brown \\
\hline 9a & White-blue \\
\hline $15 \mathrm{a}$ & Red-black \\
\hline $18 \mathrm{a}$ & Blue-white-black \\
\hline $20 \mathrm{a}$ & White-black \\
\hline $30 n$ & Black \\
\hline $31 n$ & Light blue \\
\hline $32 n$ & Yellow-green-blue-light blue \\
\hline 33a & White \\
\hline $33 n$ & Yellow-green-blue-white \\
\hline $34 n$ & Light blue-white-orange-blue \\
\hline $35 a$ & Blue \\
\hline $35 n$ & White-light blue \\
\hline $36 n$ & White-light blue \\
\hline $37 a$ & White-blue-brown-light blue \\
\hline $38 a$ & White-blue \\
\hline 39a & White \\
\hline $40 a$ & Blue-white \\
\hline $41 \mathrm{a}$ & White-black-blue \\
\hline $44 a$ & Brown \\
\hline $47 a$ & White \\
\hline $48 a$ & Green-light blue-brown-white \\
\hline 49a & Light yellow-brown \\
\hline $50 a$ & White-green \\
\hline $51 \mathrm{a}$ & Blue-black-white \\
\hline $52 a$ & Blue-white-brown-green-dark brown \\
\hline $53 a$ & White-blue-black \\
\hline $54 a$ & Blue-light blue \\
\hline $55 a$ & White-blue-black \\
\hline $57 a$ & White-light blue-pink-black \\
\hline $58 a$ & White-yellow-red-light blue \\
\hline bl & Brown \\
\hline blar & Yellow-white-blue-green \\
\hline magr & White-brown \\
\hline mapi & White-brown \\
\hline vec & Brown-white-green-light blue-light green-dark green-gray \\
\hline
\end{tabular}

Each analyzed sample was identified by one or two letters and an initial (for example $b / 9 a$ ) the first one indicating the color $(a=$ orange, $\mathrm{b}=$ white, $\mathrm{bl}=$ blue, $\mathrm{c}=$ light blue, $\mathrm{m}=$ brown, $\mathrm{n}=$ black, $\mathrm{p}=$ pink, $\mathrm{pu}=$ purple), the second one the name of the sample.

The samples were provided by the markets of antiques (flea market) in Palermo and a few samples came from private collections.

\subsection{Sampling}

To obtain a sample of composition as representative as possible of the decorations, the following precautions were taken: a) the areas for analysis were selected where a layer of colored enamel was deposited and as far as possible from other colors; b) each decoration of the same color was analyzed in three different points of the same tile to verify the reliability of the sampling method (Fig. 2).

The sampling technique, proposed by us, involved gently contacting a cotton swab soaked in hydrofluoric acid (Riedel-de Haen, Trace Select, $40 \% \mathrm{v} / \mathrm{v}$ ) with the decorated surface to chemically remove an extremely small quantity of pigment. The surface area sampled by the cotton can vary, depending on the amount of enamel that is removed. Usually about $0.20 \mathrm{~cm}^{2}$ is contacted with the cotton swab. The amount of material removed from the ceramic surface is invisible to the eye and does not cause any visible effect to the artifact. The cotton swab was then placed in a $2 \mathrm{~mL}$ test tube containing $\mathrm{HNO}_{3}(2 \% \mathrm{v} / \mathrm{v})$. The solution transferred into a small PTFE beaker was placed on a hotplate and heated to dryness. The final residue was dissolved in $1.5 \mathrm{~mL}$ of $\mathrm{HNO}_{3}$ (trace select 


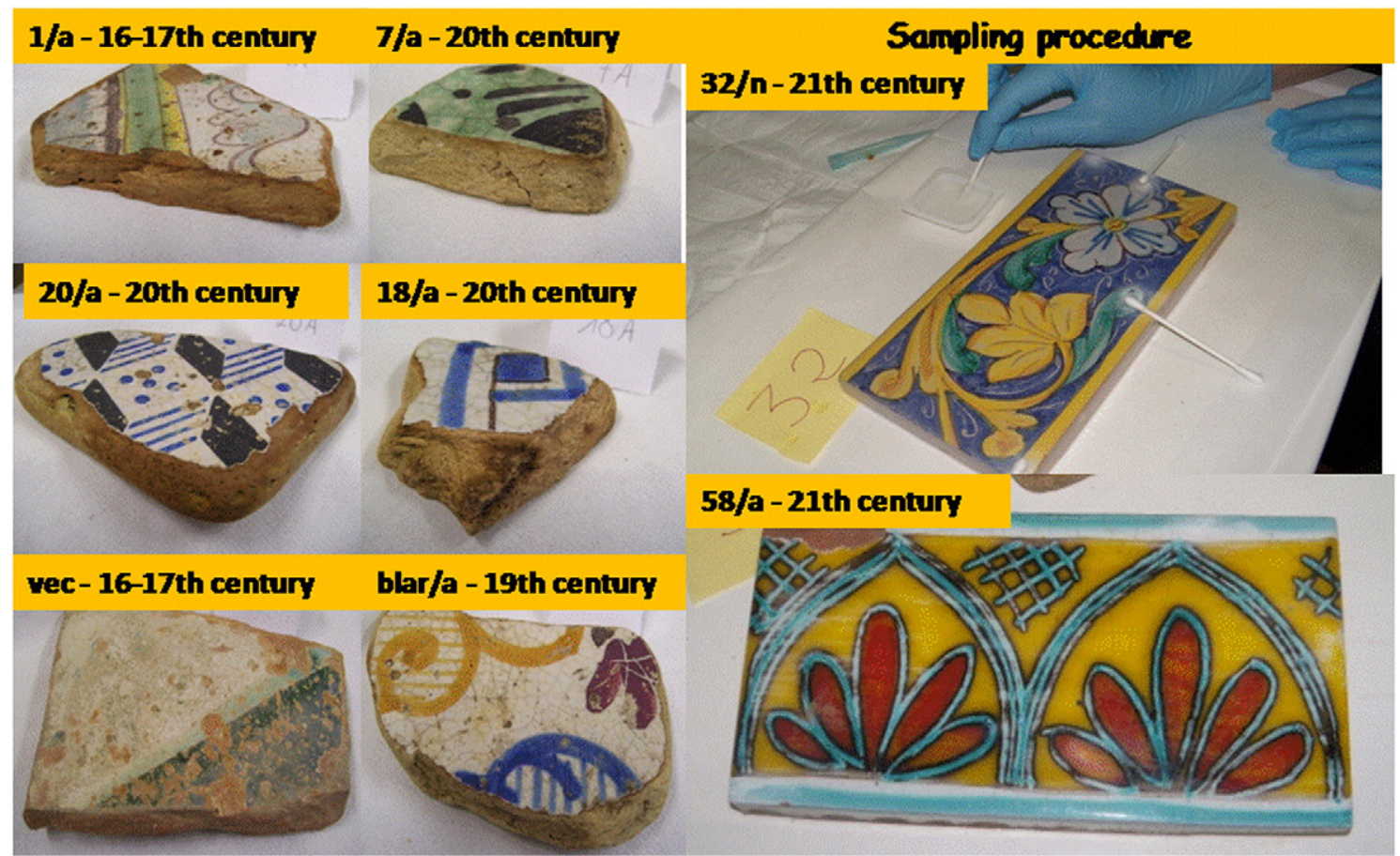

Fig. 1. Some of the studied floor tiles.

ultra for trace analysis 65\%) and the final volume was adjusted using Millipore water prior to analysis using Inductively Coupled PlasmaOptical Emission Spectrometry.

\subsection{ICP-OES analysis}

Elemental analyses were carried out on the solutions obtained from the treatment of the samples using a Perkin Elmer Optima 2100 series ICP optical emission spectrometer. The instrument is equipped with a Perkin S10 model autosampler. Data acquisition and processing were performed using the Win Lab 32 software (Perkin Elmer). Operating conditions are listed in Table 2. Twenty-four elements (Al, As, B, Be,
$\mathrm{Ba}, \mathrm{Ca}, \mathrm{Cd}, \mathrm{Co}, \mathrm{Cr}, \mathrm{Cu}, \mathrm{Fe}, \mathrm{K}, \mathrm{Mg}, \mathrm{Mn}, \mathrm{Mo}, \mathrm{Ni}, \mathrm{Pb}, \mathrm{Sb}, \mathrm{Se}, \mathrm{Si}, \mathrm{Sn}, \mathrm{Ti}, \mathrm{V}$ and $\mathrm{Zn}$ ) were determined in each sample, chosen on the basis of their significance in the study of pigments.

The ICP-OES analysis of trace elements was performed in axial viewing mode while those of major elements ( $\mathrm{Si}, \mathrm{Al}, \mathrm{K}, \mathrm{Ca}$ and $\mathrm{Pb}$ ) in radial viewing mode. The quantitative analysis was performed at two different spectral lines for each element (Table 3 ). The data of the metals reported in this paper have been calculated considering the average of the values obtained at the two wavelengths that, for all analytes, differed by less than $5 \%$. A total of 114 decorations were analyzed by about 3000 determinations of single elements.

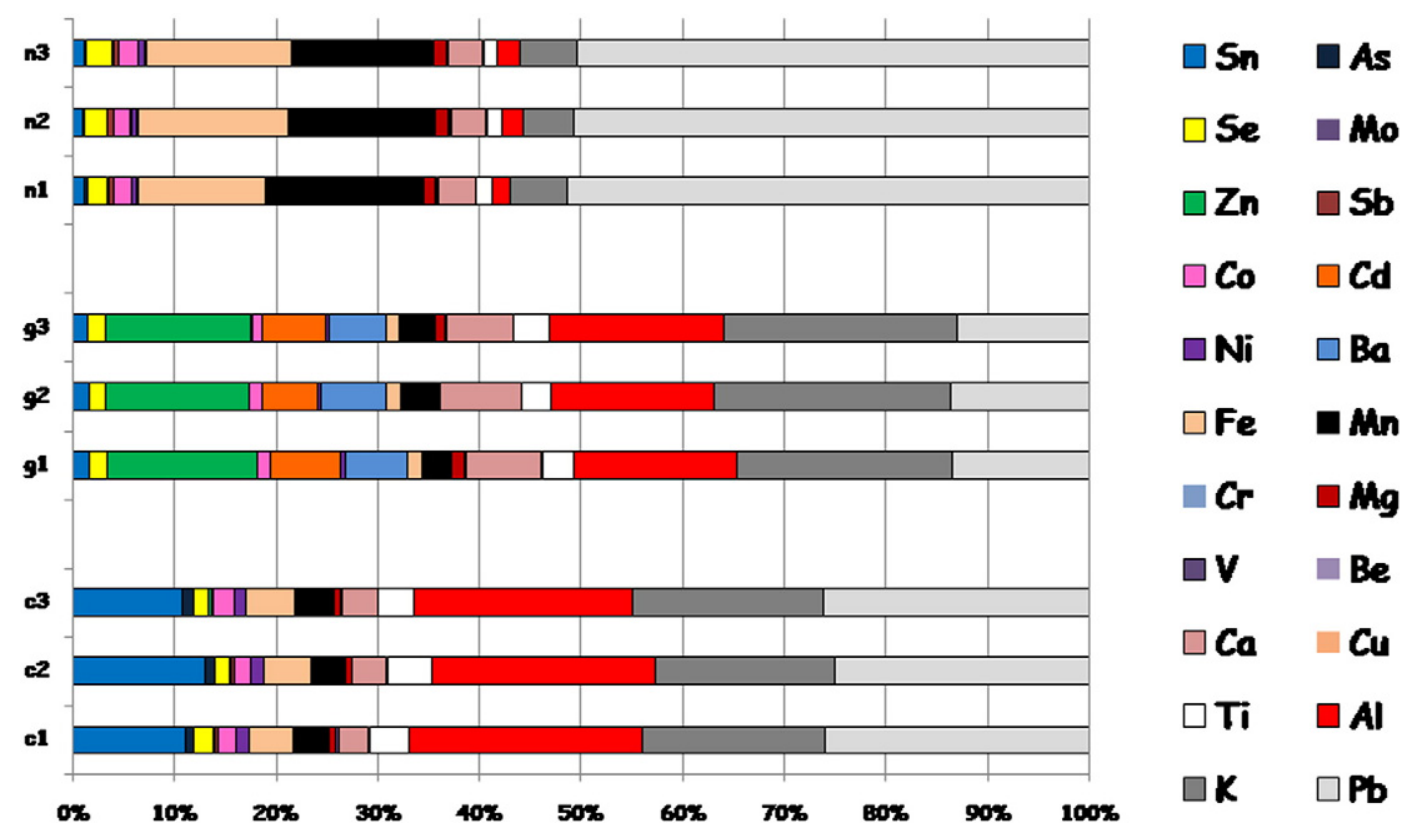

Fig. 2. Reproducibility of the sampling method. 
Table 2

ICP-OES operating conditions.

\begin{tabular}{ll}
\hline RF power $(\mathrm{W})$ & 1300 \\
Sample uptake flow rate $(\mathrm{mL} / \mathrm{min})$ & 1.5 \\
Gas flow rates $(\mathrm{L} / \mathrm{min})$ & Auxiliary: $0.2 ;$ \\
& Nebulizer: $0.8 ;$ \\
Viewing mode & Argon: 15 \\
& Axial; radial \\
\hline
\end{tabular}

\subsection{Calibration curves}

The detection limit (LOD) and the quantification limit (LOQ) were estimated as reported in previous papers [7,15] and respectively ranged from 2 to $8 \mu \mathrm{g} / \mathrm{L}$ and from 6.6 to $25 \mu \mathrm{g} / \mathrm{L}$. Calibration standard solutions were prepared by dilution with $\mathrm{HNO}_{3} 2 \%$ of a multi-element calibration standard solution (Ultra Scientific, USA, catalog number: IQC-026, Lot $n^{\circ}$ J00022) that contained 26 elements. The range of concentration of the calibration curves was between 10 and $50,000 \mu \mathrm{g} / \mathrm{L}$. For calibration, a solution of $\mathrm{HNO}_{3} 2 \%$ as blank was used. The analysis of the six standard solutions was replicated in every eight samples. Correlation coefficients of the calibration curves were in the range of 0.9985-0.9999. To eliminate memory effects related to the previous sample analysis, between two subsequent samples, a $25 \mathrm{~s}$ washing was settled. A blank was run up every eight samples. All reported data were blank corrected.

\section{Results and discussion}

To evaluate the precision of the method and in particular the feasibility of using a cotton swab for sampling glazed decorations, three replicates, sampled in different points of the same decorations (light blue c1-c3, yellow g1-g3, black n1-n3) were analyzed (Fig. 2). The relative standard deviations of the replicates on the concentrations of major metals ranged from $1 \%$ to $8 \%$ and are satisfactory for the purpose of this work.

The accuracy of analytical method was valued by analyzing samples prepared by us spiked with known amounts of some elements to solutions obtained from four samples. The recovery percentage ranged between $92 \%$ and $101 \%$.

Silicon is the most abundant element in all the samples and so its concentration cannot be useful to distinguish samples. Considering

Table 3

Wavelengths used for elemental determinations by ICP-OES.

\begin{tabular}{lll}
\hline Element & Wavelength $1(\mathrm{~nm})$ & Wavelength $2(\mathrm{~nm})$ \\
\hline $\mathrm{Al}$ & 396.153 & 308.215 \\
$\mathrm{As}$ & 193.696 & 188.979 \\
$\mathrm{~B}$ & 249.677 & 249.772 \\
$\mathrm{Ba}$ & 233.527 & 455.403 \\
$\mathrm{Be}$ & 313.107 & 313.042 \\
$\mathrm{Ca}$ & 317.933 & 315.887 \\
$\mathrm{Cd}$ & 228.802 & 214.440 \\
$\mathrm{Co}$ & 228.616 & 238.892 \\
$\mathrm{Cr}$ & 267.716 & 205.560 \\
$\mathrm{Cu}$ & 327.393 & 324.752 \\
$\mathrm{Fe}$ & 238.204 & 239.562 \\
$\mathrm{~K}$ & 766.490 & 404.721 \\
$\mathrm{Mg}$ & 285.213 & 279.077 \\
$\mathrm{Mn}$ & 257.610 & 259.372 \\
$\mathrm{Mo}$ & 202.031 & 203.845 \\
$\mathrm{Ni}$ & 221.648 \\
$\mathrm{~Pb}$ & 231.604 & 217.000 \\
$\mathrm{Sb}$ & 220.353 & 217.582 \\
$\mathrm{Se}$ & 206.836 & 203.985 \\
$\mathrm{Si}$ & 196.026 & 212.412 \\
$\mathrm{Sn}$ & 251.611 & 235.485 \\
$\mathrm{Ti}$ & 189.927 & 336.121 \\
$\mathrm{~V}$ & 334.490 & 310.230 \\
$\mathrm{Zn}$ & 292.464 & 213.857 \\
\hline
\end{tabular}

that the sampling procedure is not quantitative, to discuss and interpret the data, we normalized the concentration of the single metal, measured in the solution obtained from the sample, to that of silicon, determined within the same sampling point. Results (mean of three sampling) of chemical elemental ICP-OES analyses of the sampled material are reported in Table 4(a-i). All samples are meanly composed of $\mathrm{Si}, \mathrm{Pb}, \mathrm{Al}$ and $\mathrm{K} . \mathrm{Pb} / \mathrm{Si}$ and $\mathrm{Al} / \mathrm{Si}$ ratios, for different samples, ranged respectively from 0.114 (yellow) to 3.69 (pink) and from 0.004 (pink) to 0.160 (light blue). The relatively high amount of aluminum points to the employment of alumina silicates in vitreous coatings, in fact $\mathrm{Al} / \mathrm{Si}$ is, except for light blue and pink decorations, practically constant in all the colors (Fig. 3 ). In general, the presence of lead is compatible with the addition of a lead-tin calx to colorless enamels. The different lead to tin ratios (from 4.3 to 29.7) were probably related to the control of the viscosity and opacity of the enamels.

Also other elements, such as beryllium, boron and vanadium, have been quantified, but these have not been included in the discussion, because they did not contribute to the pigment individuation.

From the collected data, in general, we can deduce that most of the decorations associated to one color had a similar elemental composition and that the different colors could be associated with different coloring elements. In Fig. 4 the relation between the percentages of the metals and the colors of the decorations is shown.

Considering all the analyzed samples, copper is present in higher amounts in green decorations. Iron is present in higher percentages in the black, brown and yellow decorations. High amounts of $\mathrm{Ca}, \mathrm{Zn}$, $\mathrm{Sb}, \mathrm{Ti}$ and $\mathrm{Pb}$ were found mainly in pastel enamels.

The white decorations of all the tiles, except three samples (b/33n, $\mathrm{b} / 58 \mathrm{a}$ and $\mathrm{b} / 52 \mathrm{a})$, contained large amounts of $\mathrm{Pb}(\mathrm{Pb} / \mathrm{Si}=0.38), \mathrm{Sn}$ $(\mathrm{Sn} / \mathrm{Si}=0.086)$ and $\mathrm{Al}(\mathrm{Al} / \mathrm{Si}=0.086)$ and very little other elements (Fig. 5) which suggest the use of the pigments $2 \mathrm{PbCO}_{3} \cdot \mathrm{Pb}(\mathrm{OH})_{2}$ (lead white) and $\mathrm{SnO}_{2}$ (cassiterite). These compounds were used directly by themselves or mixed with other colored pigments in order to obtain different tonalities. The ratio of lead to tin in the studied tiles varies from about zero (b/33n, b/52a) to nearly 30 (b/36n). The use of different lead to tin ratios was probably related to the control of the opacity of the white areas. It should be noticed that sample $\mathrm{b} / 58 \mathrm{a}$ showed $\mathrm{Zn}$ as metal. The white zinc pigments appeared for the first time in England in 1834 [16]. The presence of this pigment in this sample indicates a decoration layer from at least the 19th century.

The presence of higher concentrations of tin $(S n / S i=1.61)$ only in sample b/33n than other white samples can be an indicator that the pigment used in this area was the compound $\mathrm{SnO}_{2}$. The presence of large concentrations of titanium $(\mathrm{Ti} / \mathrm{Si}=0.14)$ only in sample b/52a can be an indicator that the pigment used in these areas was the compound $\mathrm{TiO}_{2}$ (titanium dioxide white), while in all other samples, the $\mathrm{Ti} / \mathrm{Si}$ ratios are similar to that of the crust $\mathrm{Ti}\left(\mathrm{Ti} / \mathrm{Si}=0.023\right.$ ) [17]. The deliberate use of $\mathrm{TiO}_{2}$ as ancient pigment has not been known [18,19], its first use in objects of art came only after industrial processes were developed in the first years of 1900 for producing a pure and brilliant white. The presence of this pigment in the sample $b / 52 a$ indicates a decoration from at least the 20th century. Other white pigments were not used such as lead arsenate (since the 17th century, a more effective opacifier).

The yellow decorations, meanly, contained large amounts of $\mathrm{Pb}$ $(\mathrm{Pb} / \mathrm{Si}=0.11), \mathrm{Al}(\mathrm{Al} / \mathrm{Si}=0.090), \mathrm{Sn}(\mathrm{Sn} / \mathrm{Si}=0.027), \mathrm{Fe}(\mathrm{Fe} / \mathrm{Si}=$ 0.023 ) and very little other elements (Fig. 6), which suggests the use of lead and iron based pigments. We can suppose that the yellow enamels are made mainly of lead and iron oxides $\left(\mathrm{PbO}, \mathrm{Fe}_{2} \mathrm{O}_{3}\right.$, etc.). A significant difference concerns sample $\mathrm{g} / 58 \mathrm{a}$ contained large amounts of $\mathrm{Cd}(\mathrm{Cd} / \mathrm{Si}=0.043)$ which suggests the use of the pigments CdS.

The presence of antimony in a yellow decoration $(\mathrm{g} / 33 \mathrm{n})$ is consistent with the use of Naples Yellow $\left(\mathrm{Pb}_{2} \mathrm{Sb}_{2} \mathrm{O}_{7}\right)$. Some studies 
Table 4

Chemical composition (normalized to silicon) of the colored decorations determined by ICP-OES.

\begin{tabular}{|c|c|c|c|c|c|c|c|c|c|c|c|c|c|c|}
\hline & $\mathrm{b} / \mathrm{gr}$ & $\mathrm{b} / 9 \mathrm{a}$ & $\mathrm{b} / 1 \mathrm{a}$ & $\mathrm{b} / \mathrm{ve}$ & b/blar & $b / 34 n$ & $\mathrm{~b} / 8 \mathrm{a}$ & $\mathrm{b} / \mathrm{mp}$ & $\mathrm{b} / \mathrm{mg}$ & $\mathrm{b} / 20 \mathrm{a}$ & $\mathrm{b} / 2 \mathrm{a}$ & $b / 33 n$ & $b / 35 n$ & $\mathrm{~b} / 47 \mathrm{a}$ \\
\hline $\mathrm{Al}$ & 0.047 & 0.14 & 0.047 & 0.11 & 0.12 & 0.059 & 0.16 & 0.10 & 0.12 & 0.097 & 0.14 & 0.096 & 0.049 & 0.074 \\
\hline As & 0.0006 & 0.0007 & 0.0001 & 0.001 & 0.0012 & 0.0008 & 0.0007 & 0.0007 & 0.0009 & 0.0006 & 0.0007 & 0.0024 & 0.001 & 0.0014 \\
\hline $\mathrm{Ba}$ & 0.0002 & 0.0002 & 0.0019 & 0.0003 & 0.0037 & 0.0052 & 0.0003 & 0.0002 & 0.0002 & 0.0002 & 0.0002 & 0.018 & 0.0009 & 0.001 \\
\hline $\mathrm{Be}$ & 0.0001 & 0.0001 & 0.0001 & 0.0002 & 0.0001 & 0.0001 & 0.0002 & 0.0001 & 0.0001 & 0.0001 & 0.0001 & 0.0002 & 0.0002 & 0.0004 \\
\hline $\mathrm{Ca}$ & 0.010 & 0.0056 & 0.0095 & 0.0064 & 0.016 & 0.012 & 0.0026 & 0.0053 & 0.012 & 0.0027 & 0.0061 & 0.023 & 0.0014 & 0.015 \\
\hline $\mathrm{Cd}$ & 0.0001 & 0.0001 & 0.0028 & 0.0001 & 0.0018 & 0.002 & 0.0001 & 0.0001 & 0.0001 & 0.0001 & 0.0001 & 0.0005 & 0.0002 & 0.0003 \\
\hline Co & 0.0037 & 0.0034 & 0.0052 & 0.0064 & 0.0081 & 0.0097 & 0.0049 & 0.0040 & 0.0039 & 0.0046 & 0.0045 & 0.024 & 0.012 & 0.014 \\
\hline $\mathrm{Cr}$ & 0.0001 & 0.0001 & 0.0007 & 0.0001 & 0.001 & 0.0012 & 0.0001 & 0.0001 & 0.0001 & 0.0001 & 0.0001 & 0.0036 & 0.0002 & 0.0002 \\
\hline $\mathrm{Cu}$ & 0.0004 & 0.0003 & 0.0023 & 0.0011 & 0.0013 & 0.0013 & 0.0004 & 0.0003 & 0.0003 & 0.0003 & 0.0003 & 0.0036 & 0.0004 & 0.0002 \\
\hline $\mathrm{Fe}$ & 0.007 & 0.0076 & 0.0064 & 0.0077 & 0.012 & 0.0058 & 0.013 & 0.0074 & 0.010 & 0.012 & 0.0039 & 0.0088 & 0.0086 & 0.016 \\
\hline K & 0.036 & 0.090 & 0.069 & 0.042 & 0.069 & 0.061 & 0.064 & 0.055 & 0.058 & 0.024 & 0.036 & 0.054 & 0.021 & 0.078 \\
\hline $\mathrm{Mg}$ & 0.0029 & 0.0021 & 0.0037 & 0.0011 & 0.0053 & 0.0069 & 0.0027 & 0.0021 & 0.003 & 0.0018 & 0.0013 & 0.025 & 0.0028 & 0.006 \\
\hline $\mathrm{Mn}$ & 0.0094 & 0.0008 & 0.013 & 0.0304 & 0.019 & 0.021 & 0.0005 & 0.0099 & 0.0098 & 0.012 & 0.011 & 0.057 & 0.027 & 0.034 \\
\hline Mo & 0.0021 & 0.0038 & 0.003 & 0.0002 & 0.0053 & 0.0052 & 0.0006 & 0.0001 & 0.0001 & 0.0002 & 0.0001 & 0.0011 & 0.0005 & 0.0004 \\
\hline $\mathrm{Ni}$ & 0.002 & 0.0016 & 0.003 & 0.0019 & 0.0044 & 0.005 & 0.0016 & 0.0013 & 0.0013 & 0.0015 & 0.0014 & 0.0137 & 0.0038 & 0.0045 \\
\hline $\mathrm{Pb}$ & 0.13 & 0.070 & 0.14 & 0.067 & 0.21 & 0.12 & 0.25 & 0.31 & 0.50 & 0.33 & 0.32 & 0.01 & 0.43 & 0.54 \\
\hline $\mathrm{Sb}$ & 0.0011 & 0.0009 & 0.0017 & 0.0016 & 0.0034 & 0.0027 & 0.0001 & 0.0003 & 0.0003 & 0.0002 & 0.0004 & 0.0088 & 0.0011 & 0.0009 \\
\hline Se & 0.0044 & 0.0068 & 0.0063 & 0.0089 & 0.0095 & 0.011 & 0.0066 & 0.0053 & 0.0052 & 0.0061 & 0.0066 & 0.032 & 0.016 & 0.019 \\
\hline Sn & 0.034 & 0.035 & 0.0091 & 0.030 & 0.034 & 0.053 & 0.017 & 0.026 & 0.028 & 0.028 & 0.032 & 1.61 & 0.033 & 0.055 \\
\hline $\mathrm{Ti}$ & 0.0091 & 0.0051 & 0.0122 & 0.004 & 0.018 & 0.02 & 0.017 & 0.012 & 0.012 & 0.014 & 0.014 & 0.089 & 0.027 & 0.034 \\
\hline $\mathrm{Zn}$ & 0.0004 & 0.0011 & 0.0025 & 0.0002 & 0.0043 & 0.026 & 0.0084 & 0.0002 & 0.0002 & 0.0038 & 0.0004 & 0.064 & 0.0031 & 0.0011 \\
\hline
\end{tabular}

b

\begin{tabular}{|c|c|c|c|c|c|c|c|c|c|c|c|c|c|c|}
\hline & $\mathrm{b} / 48 \mathrm{a}$ & $\mathrm{b} / 38 \mathrm{a}$ & $\mathrm{b} / 39 \mathrm{a}$ & $\mathrm{b} / 40 \mathrm{a}$ & $\mathrm{b} / 37 \mathrm{a}$ & $b / 36 n$ & $\mathrm{~b} / 18 \mathrm{a}$ & $\mathrm{b} / 50 \mathrm{a}$ & $\mathrm{b} / 51 \mathrm{a}$ & $\mathrm{b} / 58 \mathrm{a}$ & $\mathrm{b} / 41 \mathrm{a}$ & b/ve & $\mathrm{b} / 52 \mathrm{a}$ & $\mathrm{gr} / \mathrm{vec}$ \\
\hline $\mathrm{Al}$ & 0.024 & 0.17 & 0.032 & 0.14 & 0.13 & 0.037 & 0.12 & 0.073 & 0.088 & 0.081 & 0.066 & 0.016 & 0.001 & 0.0001 \\
\hline As & 0.0071 & 0.0006 & 0.001 & 0.0016 & 0.0008 & 0.0012 & 0.0017 & 0.0011 & 0.0008 & 0.0009 & 0.0006 & 0.0014 & 0.0001 & 0.060 \\
\hline $\mathrm{Ba}$ & 0.0008 & 0.0002 & 0.0011 & 0.0003 & 0.0004 & 0.001 & 0.0009 & 0.0011 & 0.0005 & 0.025 & 0.0003 & 0.0009 & 0.0055 & 0.0076 \\
\hline $\mathrm{Be}$ & 0.0003 & 0.0001 & 0.0003 & 0.0001 & 0.0002 & 0.0004 & 0.0004 & 0.0004 & 0.0003 & 0.0003 & 0.0002 & 0.0004 & 0.0021 & 0.0002 \\
\hline $\mathrm{Ca}$ & 0.0276 & 0.0127 & 0.0256 & 0.0118 & 0.003 & 0.0066 & 0.0101 & 0.003 & 0.0055 & 0.045 & 0.0014 & 0.018 & 0.003 & 0.0001 \\
\hline $\mathrm{Cd}$ & 0.0003 & 0.0001 & 0.0002 & 0.0001 & 0.0001 & 0.0003 & 0.0003 & 0.0002 & 0.0002 & 0.0061 & 0.0001 & 0.0001 & 0.0012 & 0.0015 \\
\hline Co & 0.0108 & 0.0055 & 0.011 & 0.0049 & 0.0078 & 0.0335 & 0.0125 & 0.0109 & 0.0094 & 0.010 & 0.0057 & 0.012 & 0.066 & 0.020 \\
\hline $\mathrm{Cr}$ & 0.0002 & 0.0001 & 0.0002 & 0.0001 & 0.0001 & 0.0001 & 0.0001 & 0.0001 & 0.0001 & 0.0001 & 0.0001 & 0.0001 & 0.0001 & 0.0002 \\
\hline $\mathrm{Cu}$ & 0.0044 & 0.0003 & 0.0002 & 0.0007 & 0.0001 & 0.0002 & 0.0004 & 0.0026 & 0.0019 & 0.018 & 0.0002 & 0.0018 & 0.0001 & 0.0001 \\
\hline $\mathrm{Fe}$ & 0.027 & 0.0059 & 0.0099 & 0.0069 & 0.0042 & 0.0105 & 0.0067 & 0.021 & 0.0061 & 0.0073 & 0.0043 & 0.020 & 0.0014 & 0.047 \\
\hline $\mathrm{K}$ & 0.11 & 0.12 & 0.041 & 0.089 & 0.067 & 0.031 & 0.025 & 0.0713 & 0.0713 & 0.0573 & 0.040 & 0.059 & 0.001 & 0.066 \\
\hline $\mathrm{Mg}$ & 0.0084 & 0.0021 & 0.0062 & 0.0024 & 0.0016 & 0.0023 & 0.0021 & 0.003 & 0.0021 & 0.0056 & 0.0015 & 0.0062 & 0.0011 & 0.0002 \\
\hline $\mathrm{Mn}$ & 0.032 & 0.0008 & 0.0016 & 0.0013 & 0.0011 & 0.031 & 0.030 & 0.026 & 0.022 & 0.024 & 0.001 & 0.030 & 0.16 & 0.020 \\
\hline Mo & 0.0003 & 0.0002 & 0.0004 & 0.0001 & 0.0003 & 0.0005 & 0.0004 & 0.0003 & 0.0003 & 0.0003 & 0.0002 & 0.0004 & 0.0022 & 0.0003 \\
\hline $\mathrm{Ni}$ & 0.0038 & 0.0018 & 0.0037 & 0.0015 & 0.0025 & 0.0047 & 0.004 & 0.0035 & 0.0003 & 0.0033 & 0.0019 & 0.0040 & 0.021 & 0.0073 \\
\hline $\mathrm{Pb}$ & 1.6 & 0.35 & 0.50 & 0.42 & 0.49 & 0.63 & 0.43 & 0.35 & 0.43 & 0.025 & 0.28 & 0.71 & 0.001 & 0.84 \\
\hline $\mathrm{Sb}$ & 0.0012 & 0.0018 & 0.0008 & 0.0008 & 0.0007 & 0.0023 & 0.0009 & 0.0011 & 0.0032 & 0.0006 & 0.0004 & 0.0023 & 0.0053 & 0.0016 \\
\hline $\mathrm{Se}$ & 0.014 & 0.0076 & 0.015 & 0.0062 & 0.011 & 0.017 & 0.017 & 0.015 & 0.013 & 0.014 & 0.008 & 0.017 & 0.12 & 0.0096 \\
\hline Sn & 0.11 & 0.049 & 0.036 & 0.025 & 0.039 & 0.021 & 0.0267 & 0.039 & 0.041 & 0.044 & 0.029 & 0.051 & 0.042 & 0.026 \\
\hline $\mathrm{Ti}$ & 0.025 & 0.0076 & 0.0023 & 0.0045 & 0.0054 & 0.029 & 0.0303 & 0.0255 & 0.0226 & 0.026 & 0.0028 & 0.029 & 0.14 & 0.016 \\
\hline $\mathrm{Zn}$ & 0.0009 & 0.0011 & 0.0042 & 0.002 & 0.0025 & 0.047 & 0.0002 & 0.0002 & 0.0004 & 0.11 & 0.0004 & 0.0004 & 0.0001 & 0.0005 \\
\hline
\end{tabular}

\begin{tabular}{|c|c|c|c|c|c|c|c|c|c|c|c|c|c|c|}
\hline & $\mathrm{b} / 55 \mathrm{a}$ & $\mathrm{b} / 33 \mathrm{a}$ & $\mathrm{b} / 53 \mathrm{a}$ & $\mathrm{b} / 57 \mathrm{a}$ & $g / 1 a$ & $\mathrm{~g} / 1 \mathrm{~b}$ & $g / 32 n$ & $g / 33 n$ & g/blar & $\mathrm{g} / 58 \mathrm{a}$ & $\mathrm{gc} / 49 \mathrm{a}$ & $g / 5 a$ & $a / 34 n$ & $\mathrm{p} / 57 \mathrm{a}$ \\
\hline $\mathrm{Al}$ & 0.12 & 0.050 & 0.0439 & 0.083 & 0.09 & 0.0311 & 0.068 & 0.11 & 0.081 & 0.13 & 0.055 & 0.15 & 0.10 & 0.004 \\
\hline As & 0.0007 & 0.0004 & 0.0007 & 0.0008 & 0.0009 & 0.0014 & 0.0022 & 0.0022 & 0.0032 & 0.0007 & 0.0008 & 0.0015 & 0.0045 & 0.015 \\
\hline $\mathrm{Ba}$ & 0.0005 & 0.0004 & 0.0008 & 0.0009 & 0.0014 & 0.0027 & 0.018 & 0.0194 & 0.0008 & 0.0374 & 0.0009 & 0.0022 & 0.026 & 0.008 \\
\hline $\mathrm{Be}$ & 0.0002 & 0.0002 & 0.0003 & 0.0003 & 0.0002 & 0.0002 & 0.0005 & 0.0001 & 0.0001 & 0.0002 & 0.0003 & 0.0002 & 0.00075 & 0.004 \\
\hline $\mathrm{Ca}$ & 0.0071 & 0.002 & 0.0051 & 0.003 & 0.012 & 0.0051 & 0.034 & 0.0309 & 0.0092 & 0.0459 & 0.0002 & 0.017 & 0.050 & 0.0001 \\
\hline $\mathrm{Cd}$ & 0.0001 & 0.0001 & 0.0002 & 0.0002 & 0.0008 & 0.0017 & 0.0024 & 0.0072 & 0.002 & 0.0427 & 0.0003 & 0.0002 & 0.014 & 0.002 \\
\hline Co & 0.0076 & 0.0066 & 0.0001 & 0.0119 & 0.0033 & 0.0073 & 0.0171 & 0.023 & 0.009 & 0.0076 & 0.012 & 0.0066 & 0.068 & 0.151 \\
\hline $\mathrm{Cr}$ & 0.0002 & 0.0001 & 0.0001 & 0.0001 & 0.0001 & 0.0009 & 0.0002 & 0.0026 & 0.0011 & 0.0001 & 0.0001 & 0.0001 & 0.0079 & 0.0001 \\
\hline $\mathrm{Cu}$ & 0.0002 & 0.0002 & 0.0002 & 0.0001 & 0.0092 & 0.0041 & 0.0009 & 0.0022 & 0.0016 & 0.0004 & 0.0001 & 0.0008 & 0.0060 & 0.0001 \\
\hline $\mathrm{Fe}$ & 0.0274 & 0.0074 & 0.01 & 0.015 & 0.026 & 0.014 & 0.0065 & 0.011 & 0.060 & 0.0092 & 0.037 & 0.024 & 0.041 & 0.005 \\
\hline K & 0.053 & 0.047 & 0.037 & 0.041 & 0.072 & 0.062 & 0.037 & 0.032 & 0.053 & 0.083 & 0.038 & 0.047 & 0.0092 & 0.40 \\
\hline $\mathrm{Mg}$ & 0.003 & 0.0019 & 0.0034 & 0.0027 & 0.0035 & 0.004 & 0.0046 & 0.013 & 0.0049 & 0.0074 & 0.0013 & 0.0033 & 0.00019 & 0.0001 \\
\hline $\mathrm{Mn}$ & 0.0183 & 0.016 & 0.027 & 0.0291 & 0.0123 & 0.018 & 0.0022 & 0.044 & 0.021 & 0.018 & 0.0022 & 0.0022 & 0.14 & 0.33 \\
\hline Mo & 0.0002 & 0.0003 & 0.0004 & 0.0004 & 0.0021 & 0.0042 & 0.0006 & 0.011 & 0.005 & 0.0002 & 0.0003 & 0.0003 & 0.034 & 0.004 \\
\hline $\mathrm{Ni}$ & 0.0025 & 0.0021 & 0.0041 & 0.0038 & 0.0021 & 0.0041 & 0.0054 & 0.011 & 0.0049 & 0.0024 & 0.0038 & 0.0021 & 0.033 & 0.046 \\
\hline $\mathrm{Pb}$ & 0.73 & 0.42 & 0.39 & 0.47 & 0.15 & 0.091 & 0.089 & 0.079 & 0.068 & 0.10 & 0.24 & 0.092 & 0.28 & 3.7 \\
\hline $\mathrm{Sb}$ & 0.0006 & 0.0007 & 0.0011 & 0.0008 & 0.0052 & 0.0068 & 0.0047 & 0.030 & 0.0043 & 0.0006 & 0.0007 & 0.0097 & 0.082 & 0.01 \\
\hline $\mathrm{Se}$ & 0.010 & 0.0091 & 0.015 & 0.020 & 0.0048 & 0.0089 & 0.024 & 0.024 & 0.01 & 0.011 & 0.016 & 0.0091 & 0.074 & 0.19 \\
\hline Sn & 0.022 & 0.030 & 0.023 & 0.0247 & 0.0323 & 0.045 & 0.027 & 0.035 & 0.017 & 0.01 & 0.0155 & 0.030 & 0.036 & 0.407 \\
\hline $\mathrm{Ti}$ & 0.021 & 0.016 & 0.026 & 0.0288 & 0.0106 & 0.016 & 0.026 & 0.086 & 0.019 & 0.0194 & 0.003 & 0.022 & 0.12 & 0.292 \\
\hline $\mathrm{Zn}$ & 0.0006 & 0.0003 & 0.0027 & 0.0003 & 0.0025 & 0.0026 & 0.031 & 0.070 & 0.0042 & 0.091 & 0.0029 & 0.001 & 0.11 & 0.002 \\
\hline
\end{tabular}


Table 4 (continued)

\begin{tabular}{|c|c|c|c|c|c|c|c|c|c|c|c|c|c|c|}
\hline & $\mathrm{r} / 15 \mathrm{a}$ & $\mathrm{r} / 58 \mathrm{a}$ & c/ver & $c / 34 n$ & $c / 1 a$ & $c / 31 n$ & $c / 32 n$ & $c / 48 a$ & $c / 37 a$ & $c / 36 n$ & $c / 54 a$ & $c / 1 b$ & $c / 58 a$ & $c / 35 n$ \\
\hline Al & 0.023 & 0.12 & 1.14 & 0.12 & 0.034 & 0.088 & 0.074 & 0.012 & 0.15 & 0.047 & 0.10 & 0.087 & 0.086 & 0.050 \\
\hline As & 0.0011 & 0.0033 & 0.020 & 0.0017 & 0.004 & 0.0016 & 0.0014 & 0.078 & 0.0015 & 0.0009 & 0.0007 & 0.0033 & 0.0007 & 0.001 \\
\hline $\mathrm{Ba}$ & 0.0003 & 0.044 & 0.0009 & 0.0153 & 0.0023 & 0.0026 & 0.0085 & 0.0068 & 0.0011 & 0.0011 & 0.0027 & 0.0001 & 0.0286 & 0.001 \\
\hline $\mathrm{Be}$ & 0.0003 & 0.0002 & 0.0007 & 0.0003 & 0.0001 & 0.0002 & 0.0004 & 0.0003 & 0.0004 & 0.0004 & 0.0003 & 0.0002 & 0.0002 & 0.0004 \\
\hline $\mathrm{Ca}$ & 0.0093 & 0.033 & 0.0651 & 0.0994 & 0.0083 & 0.054 & 0.011 & 0.0083 & 0.014 & 0.0001 & 0.0079 & 0.0108 & 0.0595 & 0.0001 \\
\hline $\mathrm{Cd}$ & 0.0002 & 0.0658 & 0.0004 & 0.0048 & 0.0014 & 0.0002 & 0.0004 & 0.0018 & 0.0003 & 0.0003 & 0.0002 & 0.0001 & 0.0073 & 0.0003 \\
\hline Co & 0.0091 & 0.006 & 0.0409 & 0.0351 & 0.0075 & 0.020 & 0.0174 & 0.0209 & 0.022 & 0.017 & 0.012 & 0.0068 & 0.0069 & 0.0153 \\
\hline $\mathrm{Cr}$ & 0.032 & 0.0001 & 0.0008 & 0.0028 & 0.0008 & 0.0001 & 0.0002 & 0.0002 & 0.0002 & 0.0001 & 0.0001 & 0.0001 & 0.0002 & 0.0001 \\
\hline $\mathrm{Cu}$ & 0.0026 & 0.0018 & 0.0043 & 0.0021 & 0.010 & 0.0002 & 0.0034 & 0.0035 & 0.0001 & 0.0002 & 0.0003 & 0.0007 & 0.085 & 0.0005 \\
\hline $\mathrm{Fe}$ & 0.0124 & 0.0092 & 0.065 & 0.0099 & 0.0081 & 0.0066 & 0.0069 & 0.025 & 0.0080 & 0.0058 & 0.0103 & 0.0164 & 0.0072 & 0.0062 \\
\hline K & 0.0228 & 0.0744 & 0.54 & 0.044 & 0.068 & 0.051 & 0.040 & 0.065 & 0.080 & 0.036 & 0.071 & 0.069 & 0.075 & 0.027 \\
\hline $\mathrm{Mg}$ & 0.0044 & 0.0068 & 0.018 & 0.022 & 0.0038 & 0.0061 & 0.0039 & 0.0056 & 0.0039 & 0.0021 & 0.0028 & 0.0027 & 0.0065 & 0.0023 \\
\hline Mn & 0.0023 & 0.0144 & 0.064 & 0.048 & 0.015 & 0.0008 & 0.029 & 0.030 & 0.005 & 0.034 & 0.024 & 0.0131 & 0.016 & 0.032 \\
\hline Mo & 0.0003 & 0.0002 & 0.0007 & 0.0121 & 0.0035 & 0.0002 & 0.0004 & 0.0004 & 0.0005 & 0.0006 & 0.0003 & 0.0001 & 0.0002 & 0.0005 \\
\hline $\mathrm{Ni}$ & 0.0029 & 0.0019 & 0.015 & 0.0117 & 0.0059 & 0.0022 & 0.0041 & 0.0085 & 0.0053 & 0.0047 & 0.0031 & 0.0048 & 0.0023 & 0.0044 \\
\hline $\mathrm{Pb}$ & 0.063 & 0.15 & 0.22 & 0.26 & 0.11 & 0.25 & 0.23 & 0.57 & 0.71 & 0.28 & 0.45 & 0.098 & 0.023 & 0.25 \\
\hline $\mathrm{Sb}$ & 0.0013 & 0.0004 & 0.0064 & 0.0062 & 0.0021 & 0.0004 & 0.0009 & 0.0012 & 0.0013 & 0.0013 & 0.0014 & 0.0009 & 0.0004 & 0.0014 \\
\hline Se & 0.012 & 0.0104 & 0.029 & 0.026 & 0.0073 & 0.0093 & 0.0184 & 0.0158 & 0.0216 & 0.020 & 0.013 & 0.0072 & 0.0095 & 0.019 \\
\hline Sn & 0.065 & 0.0052 & 0.32 & 0.0001 & 0.045 & 0.001 & 0.020 & 0.043 & 0.0349 & 0.030 & 0.025 & 0.042 & 0.043 & 0.027 \\
\hline $\mathrm{Ti}$ & 0.014 & 0.016 & 0.076 & 0.044 & 0.014 & 0.0037 & 0.017 & 0.026 & 0.0055 & 0.033 & 0.023 & 0.015 & 0.021 & 0.0314 \\
\hline $\mathrm{Zn}$ & 0.0009 & 0.0936 & 0.0036 & 0.15 & 0.010 & 0.072 & 0.048 & 0.0008 & 0.0079 & 0.0068 & 0.0041 & 0.0008 & 0.14 & 0.0048 \\
\hline
\end{tabular}

\begin{tabular}{|c|c|c|c|c|c|c|c|c|c|c|c|c|c|c|}
\hline & $c / 57 a$ & $\mathrm{v} / \mathrm{ve}$ & $\mathrm{v} / 1 \mathrm{a}$ & $\mathrm{v} / 1 \mathrm{~b}$ & $\mathrm{v} / 7 \mathrm{a}$ & $\mathrm{v} / 8 \mathrm{a}$ & v/blar & $v / 33 n$ & $\mathrm{v} / 32 \mathrm{n}$ & $\mathrm{v} / 52 \mathrm{a}$ & $\mathrm{v} / 50 \mathrm{a}$ & $\mathrm{v} / 48 \mathrm{a}$ & $\mathrm{vc} / \mathrm{vec}$ & vs/vec \\
\hline $\mathrm{Al}$ & 0.10 & 0.090 & 0.096 & 0.075 & 0.14 & 0.14 & 0.072 & 0.047 & 0.054 & 0.080 & 0.053 & 0.023 & 0.053 & 0.033 \\
\hline As & 0.0025 & 0.004 & 0.0007 & 0.0002 & 0.0013 & 0.0005 & 0.0052 & 0.0013 & 0.0013 & 0.0026 & 0.0013 & 0.002 & 0.0036 & 0.0352 \\
\hline $\mathrm{Ba}$ & 0.0014 & 0.0023 & 0.001 & 0.0028 & 0.003 & 0.006 & 0.0008 & 0.0153 & 0.0186 & 0.0012 & 0.0009 & 0.0007 & 0.001 & 0.0143 \\
\hline $\mathrm{Be}$ & 0.0006 & 0.0001 & 0.0001 & 0.0001 & 0.0001 & 0.0001 & 0.0002 & 0.0001 & 0.0003 & 0.0005 & 0.0002 & 0.0003 & 0.0004 & 0.0004 \\
\hline $\mathrm{Ca}$ & 0.0053 & 0.0079 & 0.025 & 0.0067 & 0.0153 & 0.0074 & 0.019 & 0.030 & 0.0445 & 0.0049 & 0.0111 & 0.016 & 0.028 & 0.0491 \\
\hline $\mathrm{Cd}$ & 0.0004 & 0.0001 & 0.0006 & 0.0017 & 0.0018 & 0.0001 & 0.0024 & 0.0036 & 0.0009 & 0.0003 & 0.0001 & 0.0002 & 0.0003 & 0.0013 \\
\hline Co & 0.0235 & 0.0069 & 0.0027 & 0.0077 & 0.0079 & 0.0082 & 0.012 & 0.016 & 0.012 & 0.017 & 0.0082 & 0.0114 & 0.013 & 0.030 \\
\hline $\mathrm{Cr}$ & 0.0002 & 0.0001 & 0.0001 & 0.0012 & 0.0011 & 0.0009 & 0.0037 & 0.0021 & 0.0002 & 0.0001 & 0.0001 & 0.0002 & 0.0002 & 0.0002 \\
\hline $\mathrm{Cu}$ & 0.0001 & 0.0165 & 0.039 & 0.059 & 0.038 & 0.0049 & 0.0036 & 0.23 & 0.14 & 0.023 & 0.050 & 0.037 & 0.049 & 0.020 \\
\hline $\mathrm{Fe}$ & 0.013 & 0.0164 & 0.022 & 0.022 & 0.0105 & 0.012 & 0.022 & 0.028 & 0.0049 & 0.013 & 0.024 & 0.026 & 0.062 & 0.0123 \\
\hline K & 0.04 & 0.050 & 0.084 & 0.059 & 0.046 & 0.047 & 0.12 & 0.020 & 0.032 & 0.041 & 0.079 & 0.062 & 0.078 & 0.099 \\
\hline $\mathrm{Mg}$ & 0.0046 & 0.002 & 0.0051 & 0.0066 & 0.0062 & 0.0027 & 0.0079 & 0.01 & 0.0053 & 0.0029 & 0.0041 & 0.0062 & 0.0094 & 0.019 \\
\hline Mn & 0.046 & 0.013 & 0.0069 & 0.018 & 0.019 & 0.0085 & 0.025 & 0.037 & 0.0039 & 0.043 & 0.032 & 0.033 & 0.032 & 0.0391 \\
\hline Mo & 0.0006 & 0.0001 & 0.0016 & 0.0044 & 0.0046 & 0.0002 & 0.0003 & 0.0091 & 0.0004 & 0.0005 & 0.0002 & 0.0003 & 0.0002 & 0.0005 \\
\hline $\mathrm{Ni}$ & 0.0064 & 0.0027 & 0.0016 & 0.0045 & 0.0053 & 0.0011 & 0.0034 & 0.009 & 0.0039 & 0.0051 & 0.0026 & 0.0038 & 0.0042 & 0.01 \\
\hline $\mathrm{Pb}$ & 0.76 & 0.063 & 0.33 & 0.16 & 0.19 & 0.59 & 0.099 & 0.0049 & 0.015 & 0.50 & 0.38 & 1.1 & 0.52 & 1.1 \\
\hline $\mathrm{Sb}$ & 0.0015 & 0.0078 & 0.0012 & 0.0033 & 0.0035 & 0.0056 & 0.0063 & 0.0048 & 0.0016 & 0.0028 & 0.0023 & 0.001 & 0.0034 & 0.0079 \\
\hline Se & 0.027 & 0.0051 & 0.0036 & 0.0093 & 0.0094 & 0.0045 & 0.012 & 0.021 & 0.017 & 0.022 & 0.011 & 0.0167 & 0.016 & 0.019 \\
\hline Sn & 0.0346 & 0.035 & 0.0091 & 0.021 & 0.043 & 0.019 & 0.27 & 0.034 & 0.030 & 0.17 & 0.036 & 0.079 & 0.051 & 0.066 \\
\hline $\mathrm{Ti}$ & 0.045 & 0.011 & 0.0095 & 0.019 & 0.0204 & 0.013 & 0.021 & 0.085 & 0.044 & 0.036 & 0.0193 & 0.027 & 0.038 & 0.037 \\
\hline $\mathrm{Zn}$ & 0.0001 & 0.0013 & 0.0014 & 0.005 & 0.006 & 0.0026 & 0.0064 & 0.051 & 0.035 & 0.0005 & 0.0014 & 0.0004 & 0.0016 & 0.0017 \\
\hline
\end{tabular}

\begin{tabular}{|c|c|c|c|c|c|c|c|c|c|c|c|c|c|c|}
\hline & $\mathrm{bl} / 2 \mathrm{a}$ & $b l / 32 n$ & $b l / 35 n$ & $\mathrm{bl} / 18 \mathrm{a}$ & $b l / 33 n$ & $\mathrm{bl} / 40 \mathrm{a}$ & $\mathrm{bl} / 51 \mathrm{a}$ & $\mathrm{bl} / 41 \mathrm{a}$ & $\mathrm{bl} / 9 \mathrm{a}$ & $\mathrm{bl} / \mathrm{blar}$ & $b l / 34 n$ & $\mathrm{bl} / 37 \mathrm{a}$ & $\mathrm{bl} / 38 \mathrm{a}$ & $\mathrm{bl} / 54 \mathrm{a}$ \\
\hline $\mathrm{Al}$ & 0.093 & 0.056 & 0.032 & 0.15 & 0.061 & 0.083 & 0.055 & 0.001 & 0.15 & 0.11 & 0.0057 & 0.12 & 0.18 & 0.075 \\
\hline As & 0.0008 & 0.0016 & 0.0008 & 0.0077 & 0.0016 & 0.0066 & 0.003 & 0.0051 & 0.0005 & 0.0097 & 0.0003 & 0.0008 & 0.0005 & 0.0009 \\
\hline $\mathrm{Ba}$ & 0.0024 & 0.014 & 0.0009 & 0.0003 & 0.018 & 0.0021 & 0.0004 & 0.004 & 0.0002 & 0.001 & 0.0015 & 0.0006 & 0.0004 & 0.0041 \\
\hline $\mathrm{Be}$ & 0.0001 & 0.0001 & 0.0003 & 0.0002 & 0.0001 & 0.0004 & 0.0002 & 0.0018 & 0.0001 & 0.0001 & 0.0001 & 0.0003 & 0.0001 & 0.0002 \\
\hline $\mathrm{Ca}$ & 0.031 & 0.031 & 0.0035 & 0.0096 & 0.024 & 0.018 & 0.0051 & 0.0003 & 0.0044 & 0.010 & 0.003 & 0.0023 & 0.011 & 0.015 \\
\hline $\mathrm{Cd}$ & 0.0001 & 0.0004 & 0.0003 & 0.0003 & 0.0055 & 0.0004 & 0.0001 & 0.0012 & 0.0052 & 0.0008 & 0.0008 & 0.0002 & 0.0001 & 0.0001 \\
\hline Co & 0.0354 & 0.0323 & 0.0233 & 0.023 & 0.070 & 0.032 & 0.017 & 0.17 & 0.0202 & 0.022 & 0.0086 & 0.032 & 0.022 & 0.0236 \\
\hline $\mathrm{Cr}$ & 0.0001 & 0.0002 & 0.0001 & 0.0001 & 0.0031 & 0.0002 & 0.0001 & 0.001 & 0.0001 & 0.0001 & 0.0005 & 0.0001 & 0.0001 & 0.0001 \\
\hline $\mathrm{Cu}$ & 0.0007 & 0.0019 & 0.0003 & 0.0007 & 0.0032 & 0.0011 & 0.0031 & 0.0002 & 0.0004 & 0.0009 & 0.0004 & 0.0002 & 0.0002 & 0.0004 \\
\hline $\mathrm{Fe}$ & 0.0097 & 0.0106 & 0.009 & 0.011 & 0.0067 & 0.0107 & 0.0077 & 0.0006 & 0.0091 & 0.015 & 0.0011 & 0.004 & 0.0061 & 0.012 \\
\hline K & 0.047 & 0.041 & 0.024 & 0.044 & 0.055 & 0.076 & 0.045 & 0.14 & 0.068 & 0.062 & 0.013 & 0.055 & 0.088 & 0.061 \\
\hline $\mathrm{Mg}$ & 0.0043 & 0.0054 & 0.0025 & 0.0027 & 0.0143 & 0.005 & 0.0025 & 0.001 & 0.0018 & 0.0032 & 0.0022 & 0.0013 & 0.0022 & 0.0027 \\
\hline $\mathrm{Mn}$ & 0.0113 & 0.032 & 0.029 & 0.015 & 0.056 & 0.0024 & 0.017 & 0.0059 & 0.0011 & 0.0066 & 0.0084 & 0.0013 & 0.0007 & 0.015 \\
\hline Mo & 0.0001 & 0.0082 & 0.0005 & 0.0003 & 0.0141 & 0.0004 & 0.0002 & 0.002 & 0.0001 & 0.0001 & 0.0021 & 0.0003 & 0.0001 & 0.0001 \\
\hline $\mathrm{Ni}$ & 0.0027 & 0.0079 & 0.0043 & 0.0025 & 0.014 & 0.0054 & 0.0029 & 0.018 & 0.0014 & 0.0032 & 0.002 & 0.004 & 0.0015 & 0.0024 \\
\hline $\mathrm{Pb}$ & 0.37 & 0.031 & 0.32 & 0.54 & 0.026 & 0.71 & 0.39 & 1.7 & 0.055 & 0.14 & 0.015 & 0.43 & 0.59 & 0.43 \\
\hline $\mathrm{Sb}$ & 0.0009 & 0.001 & 0.0018 & 0.0008 & 0.0076 & 0.0019 & 0.0057 & 0.0063 & 0.0006 & 0.0014 & 0.0011 & 0.0008 & 0.0012 & 0.0015 \\
\hline $\mathrm{Se}$ & 0.0061 & 0.0187 & 0.017 & 0.008 & 0.032 & 0.019 & 0.0092 & 0.077 & 0.0053 & 0.0034 & 0.0045 & 0.013 & 0.0064 & 0.0081 \\
\hline Sn & 0.037 & 0.033 & 0.020 & 0.025 & 0.040 & 0.018 & 0.034 & 0.12 & 0.019 & 0.0265 & 0.0067 & 0.025 & 0.0236 & 0.027 \\
\hline $\mathrm{Ti}$ & 0.013 & 0.0466 & 0.028 & 0.016 & 0.11 & 0.0029 & 0.017 & 0.0006 & 0.0057 & 0.0091 & 0.0074 & 0.0061 & 0.0074 & 0.036 \\
\hline $\mathrm{Zn}$ & 0.0072 & 0.078 & 0.025 & 0.0003 & 0.16 & 0.0001 & 0.0003 & 0.094 & 0.0013 & 0.0025 & 0.0091 & 0.050 & 0.0004 & 0.030 \\
\hline
\end{tabular}

\begin{tabular}{|c|c|c|c|c|c|c|c|c|c|c|c|c|c|c|}
\hline & $\mathrm{bl} / 53 \mathrm{a}$ & $\mathrm{bl} / 52 \mathrm{a}$ & $\mathrm{bl} / 55 \mathrm{a}$ & $\mathrm{bl} / 35 \mathrm{a}$ & $\mathrm{m} / 1 \mathrm{~b}$ & $\mathrm{~m} / 1 \mathrm{bb}$ & $\mathrm{m} / 1 \mathrm{a}$ & $\mathrm{m} / \mathrm{bl}$ & $\mathrm{m} / 5 \mathrm{a}$ & $\mathrm{m} / \mathrm{ve}$ & $\mathrm{m} / 8 \mathrm{a}$ & $\mathrm{m} / \mathrm{map}$ & $\mathrm{m} / 44 \mathrm{a}$ & $\mathrm{m} / \mathrm{mgr}$ \\
\hline $\mathrm{Al}$ & 0.044 & 0.06 & 0.12 & 0.033 & 0.087 & 0.11 & 0.078 & 0.13 & 0.11 & 0.062 & 0.12 & 0.069 & 0.11 & 0.059 \\
\hline
\end{tabular}


Table 4 (continued)

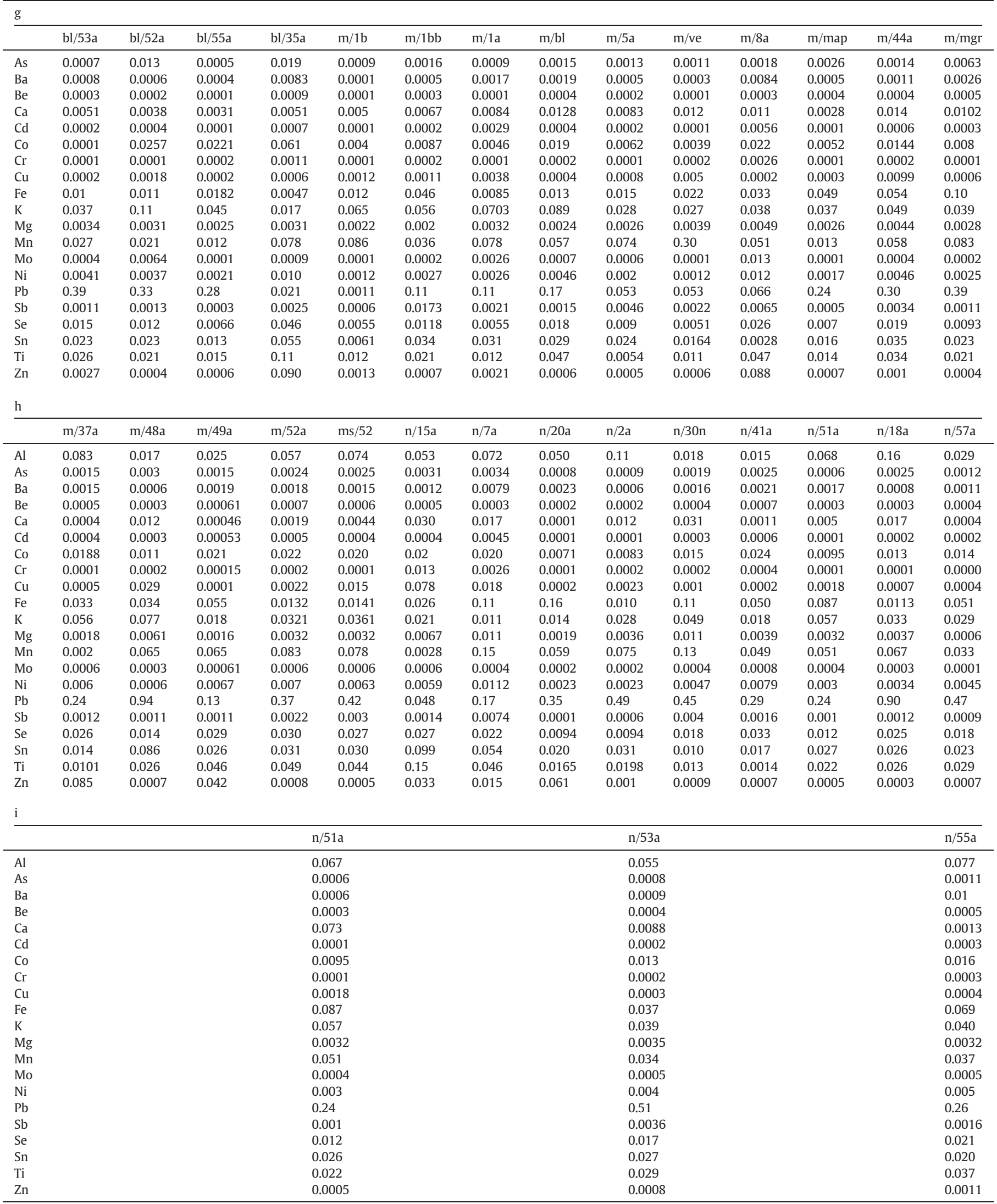

regarding Naples Yellow have been published with particular attention given to the identification of the three elements $(\mathrm{Pb}, \mathrm{Sb}, \mathrm{Sn})$ in oil painting and ceramic [20-22].
In samples g/32n and g33/n (Fig. 6), produced in the same ceramic laboratory (located at Santo Stefano di Camastra) around the year 2000 , the selenium content, equal in the two tiles and slightly more 


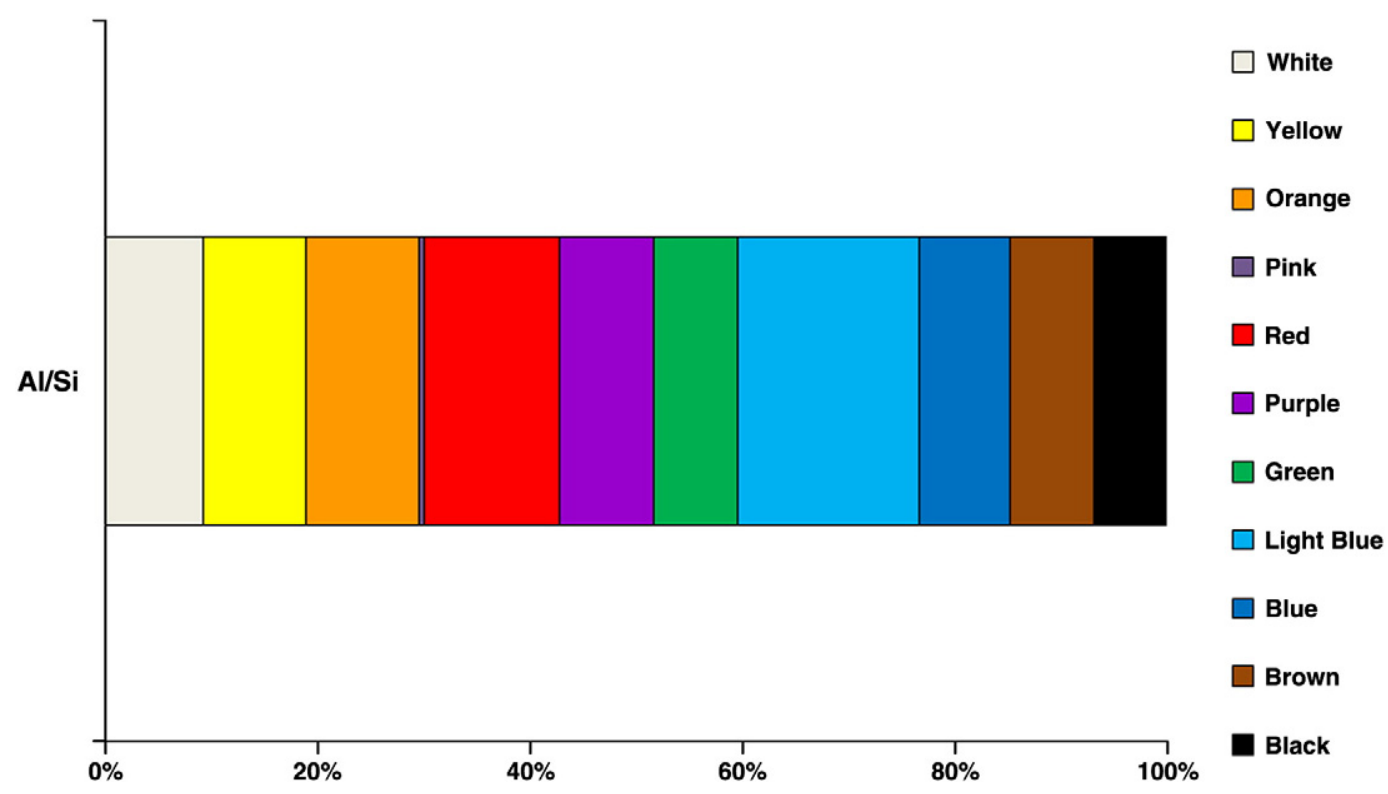

Fig. 3. AI/Si ratios in the different decorations.

abundant as compared to the other yellow samples, may be attributed to the use of a sulfo-selenide. This class of substances did not become commercially available as pigment until about the first quarter of the 20th century [23]. Traces of selenium present in all other samples can be attributed to impurities of the materials used in the preparation of pigments.

In samples $\mathrm{g} 32 / \mathrm{n}$ and $\mathrm{g} 33 / \mathrm{n}$, the concentration of titanium $(\mathrm{Ti} / \mathrm{Si}=$ 0.026 and 0.086 ), significantly higher as compared to all other samples, might be due to the use of $\mathrm{TiO}_{2}$ to obtain the desired pastel shades. As in the case of blue samples (bl32/n and bl33/n), the compositions of the yellow decorations of the two tiles, produced in the same laboratory, are very similar.

In light yellow decoration $\mathrm{gc} / 49 \mathrm{a}$, where Fe is the principal element of coloring pigment, the concentration of $\mathrm{Pb}$ is much higher $(\mathrm{Pb} / \mathrm{Si}=0.24)$ than that in dark yellows (usually $\mathrm{Pb} / \mathrm{Si}=0.11$ ). In this case the light yellow color was obtained by adding white lead to an iron oxide (for example Yellow Mars). Considering the absence of antimony, we can exclude the use of Naples Yellow.

The light blue tile decorations contain opacifiers based on lead, aluminum and potassium whose amounts, normalized to silicon, respectively are $0.32,0.16$ and 0.093 and are characterized by presence of cobalt $(\mathrm{Co} / \mathrm{Si}=0.019)$ and very little $\mathrm{Cu}(\mathrm{Cu} / \mathrm{Si}=0.0085)$ (Fig. 7). The pigment added in this case probably was smalt, a mixture containing cobalt oxide (zaffre or zaffera).

Although cobalt was isolated in the 18th century, its use (in the form of compounds or of ores) as pigment has been known for thousands of years because, even at low percentage, it produces a noticeable tint in an enamel [24]. Nearly all enamels develop a blue color with cobalt compounds, even if in some conditions a pink one will develop as in sample $\mathrm{p} / 57 \mathrm{a}$ in which the $\mathrm{Co} / \mathrm{Si}$ ratio is much higher $(0.15)$ compared

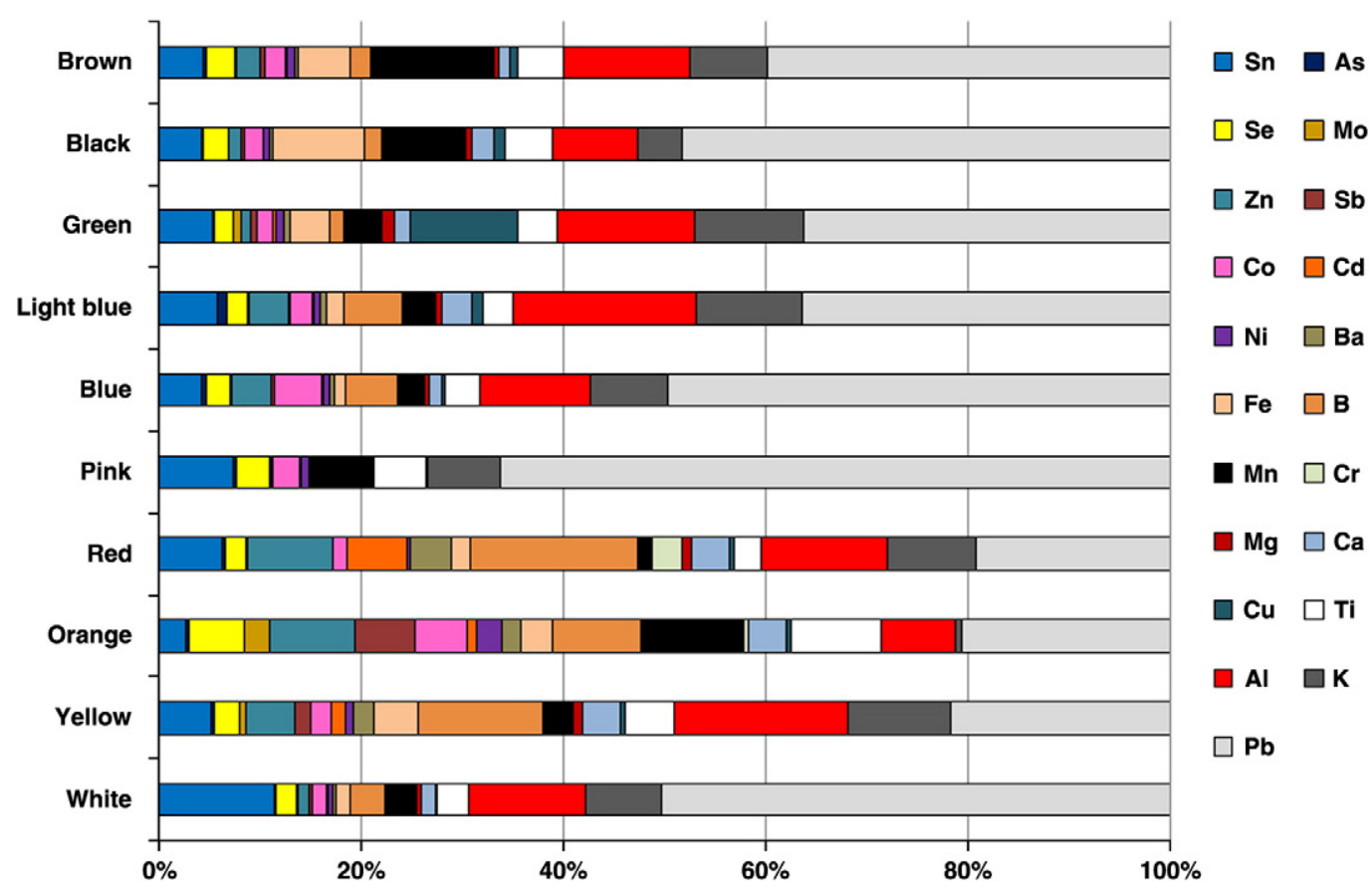

Fig. 4. Chemical composition of the colored decorations. 


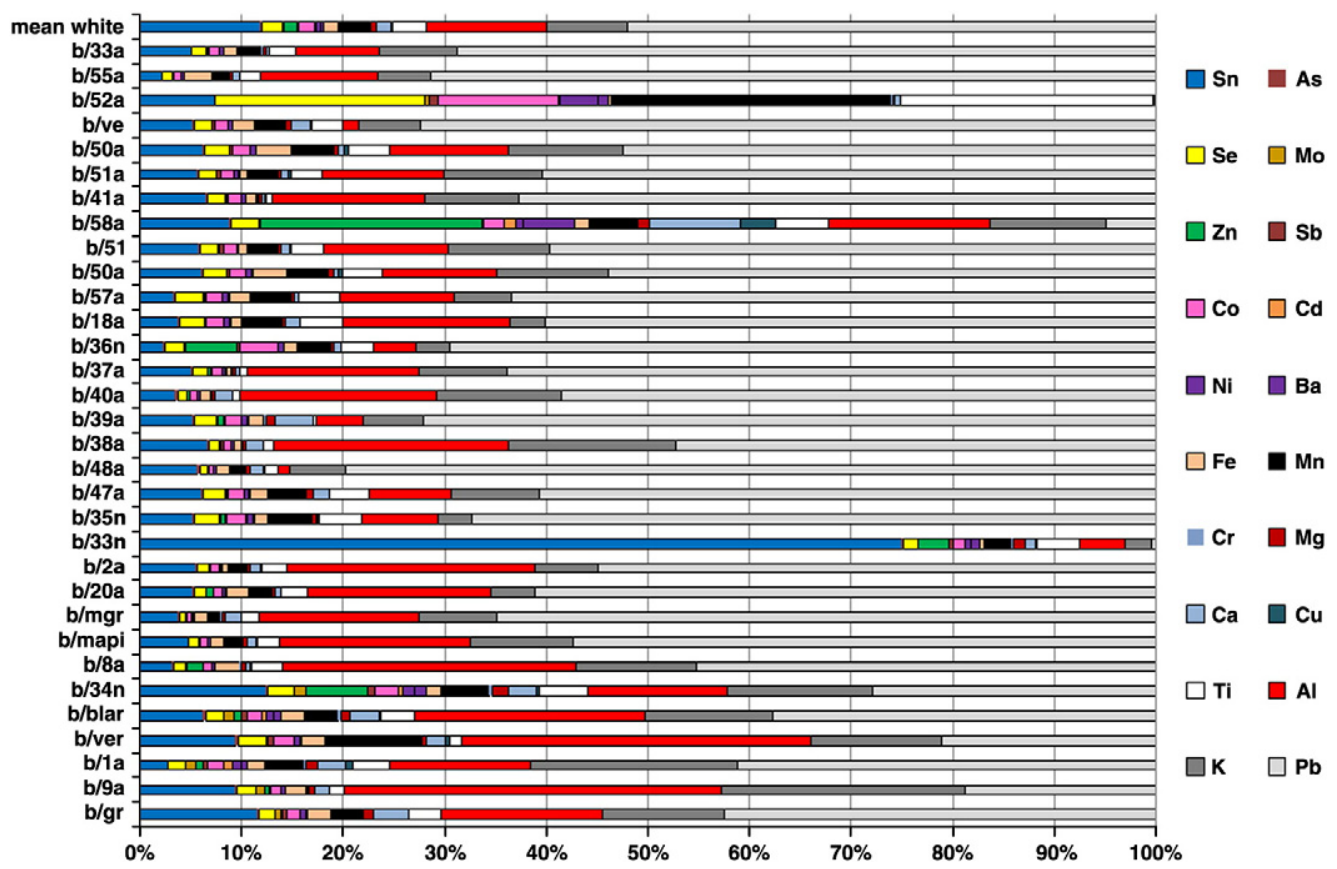

Fig. 5. Chemical composition of the white decorations.

to all the other samples with the exception of a single blue decoration (bl/41a). Several studies investigated the nature and origin of the cobalt minerals used to obtain deep blue pigments and are based on analysis of the elements associated with element $[25,26]$.

Samples c58/a and c48/a differ from the other enamels because of their different composition. In sample c58/a, the concentration of copper, significantly higher $(\mathrm{Cu} / \mathrm{Si}=0.085)$ as compared to all other samples, might be due to the use of pigments containing copper $\left(\mathrm{CuO}, 2 \mathrm{CuCO}_{3} \cdot \mathrm{Cu}(\mathrm{OH})_{2}\right.$, chrysocolla, etc.) for its coloration, although the zinc $(\mathrm{Zn} / \mathrm{Si}=0.14)$ and barium $(\mathrm{Ba} / \mathrm{Si}=0.029)$ contents appear to be somewhat high. The last elements, as white zinc $(\mathrm{ZnO})$ or white fixed $\left(\mathrm{BaSO}_{4}\right)$, may have been used to obtain the desired hue. In sample c48/a, the concentrations of arsenic $(\mathrm{As} / \mathrm{Si}=0.078)$, significantly higher as compared to all other samples (meanly $\mathrm{As} / \mathrm{Si}=$ $0.0090)$, might be due to the use of orpiment $\left(\mathrm{As}_{2} \mathrm{~S}_{3}\right)$ for its yellow coloration to obtain a greenish hue. The absence of copper excludes the use of green Scheele $\left(\mathrm{CuHAsO}_{3}\right)$. To our present knowledge, no data are published on the use of copper compounds in blue enamels.

The concomitant presence of $\mathrm{Fe}, \mathrm{Ni}, \mathrm{Mn}$ and As in our light blue samples is due to the fact that the pigments were most likely obtained by materials containing these elements. In particular, it is quite interesting to consider $\mathrm{Co} / \mathrm{As}$ ratio values in order to better understand the

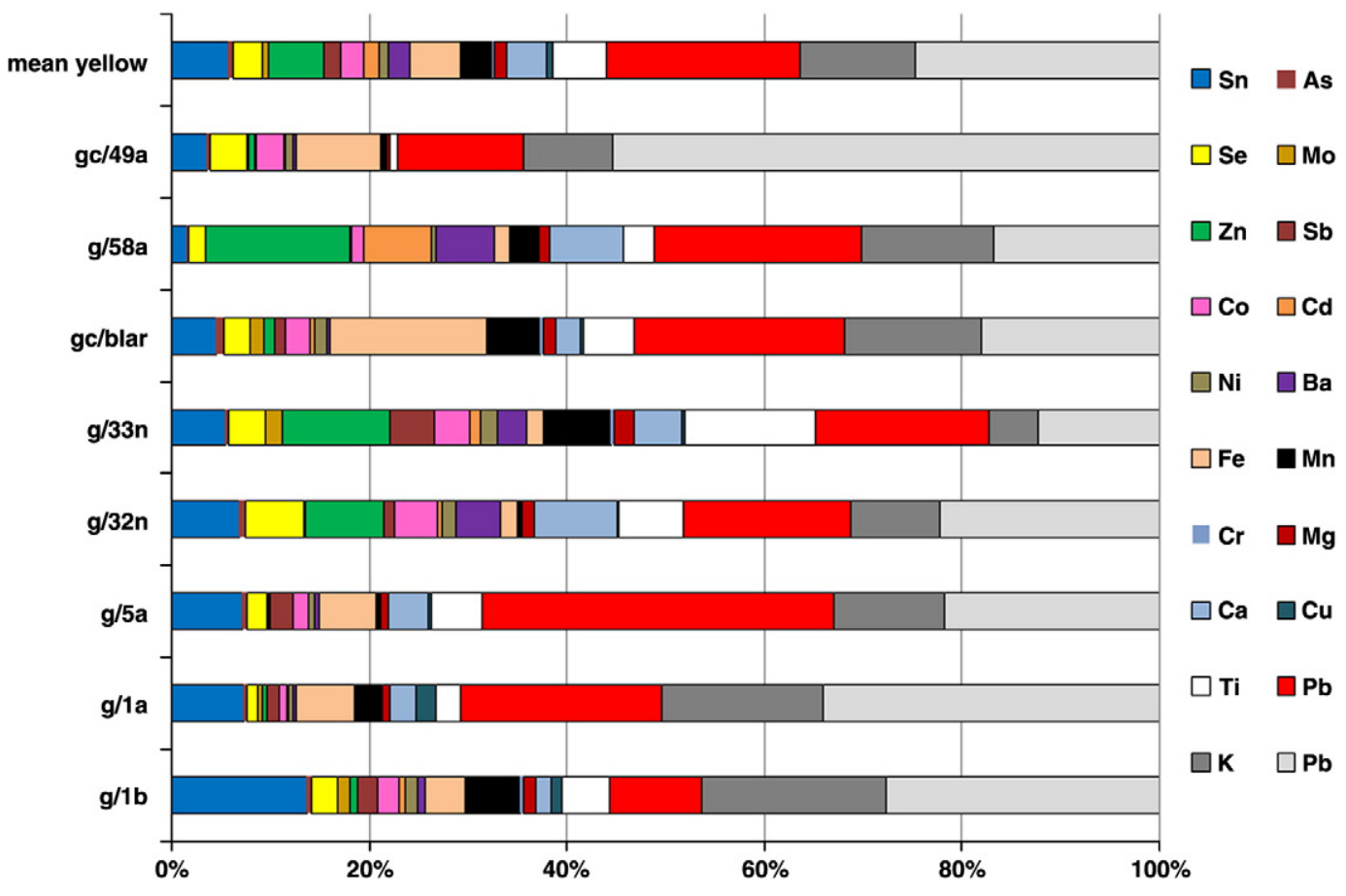

Fig. 6. Chemical composition of the yellow decorations. 


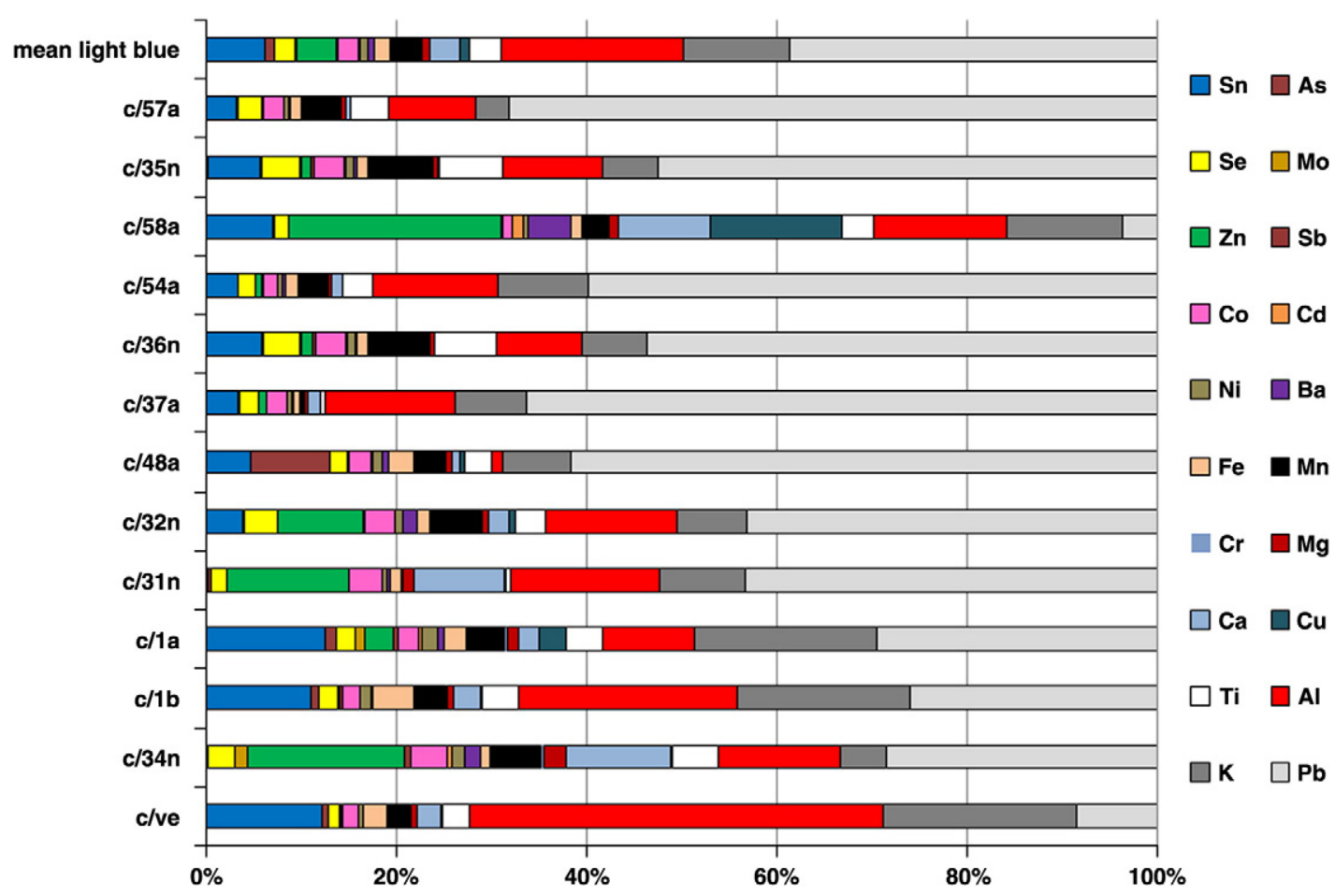

Fig. 7. Chemical composition of the light blue decorations.

technology used for the pigment preparation. Considering nine light blue samples, meanly, the high ratio $(\mathrm{Co} / \mathrm{As}=13.6)$ indicates that the firing conditions used during the production process were strong enough (oxidizing atmosphere and high temperature) to promote arsenic volatilization. In particular, we note that in very old samples (c/1a, c/1b e c/48a) ratios Co/As are very low (from 0.9 to 2.3). This confirms that the cobalt-containing pigments were not prepared or utilized in a sufficiently oxidizing environment to remove the arsenic.

Most of the blue decorations are characterized by relatively high cobalt $(\mathrm{Co} / \mathrm{Si}=0.035)$ and tin $(\mathrm{Sn} / \mathrm{Si}=0.032)$ contents. The blue color of the decorations can be ascribed to the use of $\mathrm{CoO} \cdot \mathrm{nSnO}_{2}+$ $\mathrm{CoSn}(\mathrm{OH})_{6}$ (Blue Cerulean) chromophore of high coloration power. These results are in good agreement with what several authors [27] refer for dark blue enamels, recommend saffre ( $\mathrm{CoO}+$ contaminant) or smalt $\left(\mathrm{SiO}_{2}-\mathrm{K}_{2} \mathrm{O}-\mathrm{CoO}+\right.$ contaminants $)$ as coloring substance. From the 19th century, the authors prescribe pure industrial produced $\mathrm{CoO}$ [27]. Only Neri [27] suggested in some blue enamel the use of $\mathrm{CuO}$.

The significant presence of molybdenum in only three blue samples ( $\mathrm{bl} / 34 \mathrm{n}, \mathrm{bl} / 33 \mathrm{n}$ and $\mathrm{bl} / 32 \mathrm{n}$ ) can be an indication that the pigment

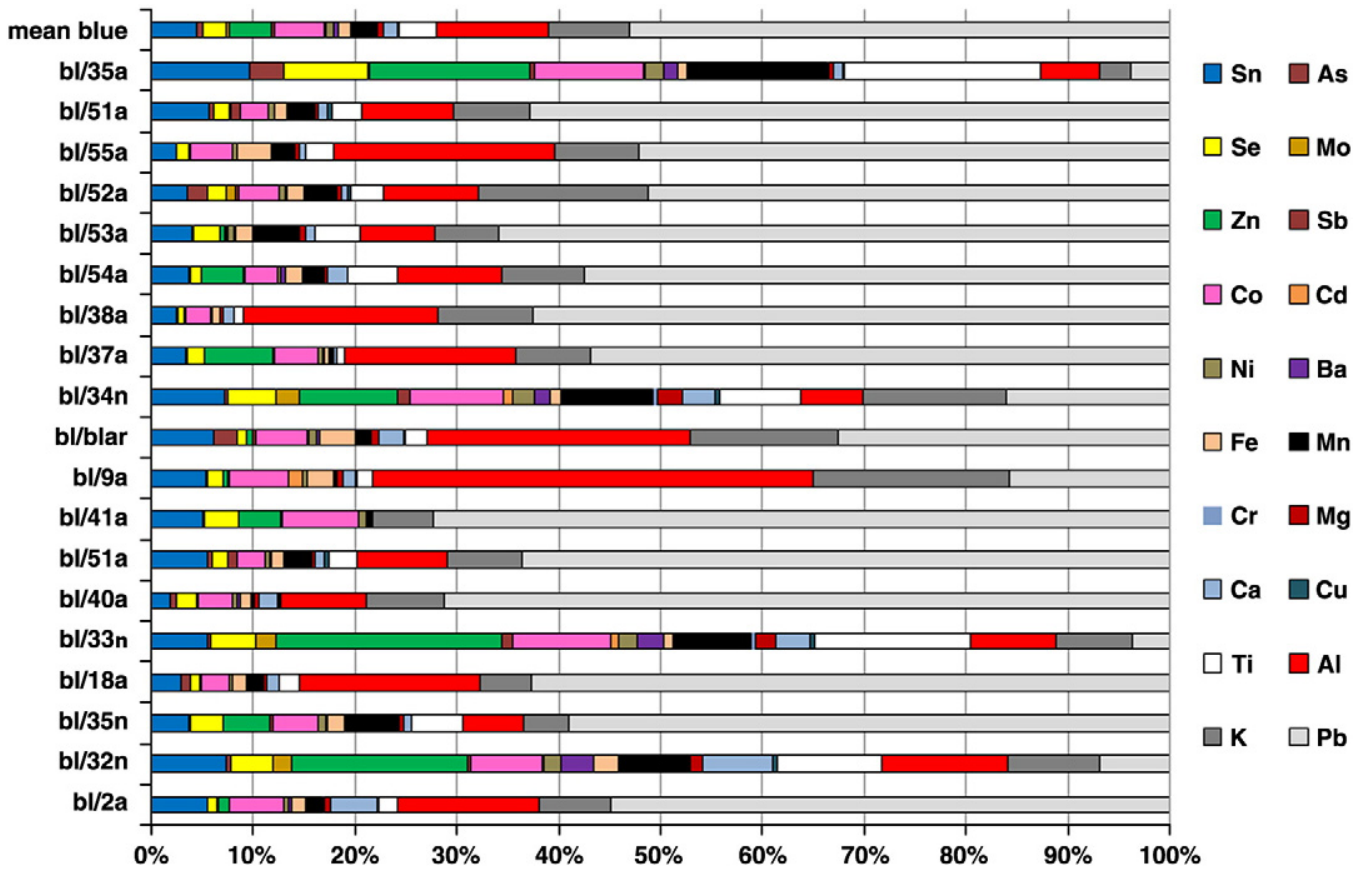

Fig. 8. Chemical composition of the blue decorations. 
used in these areas was a compound termed molybdenum blue [28]. It's interesting to note that, for the same color of the decoration, tiles produced in the same geographic area $(\mathrm{bl} / 34 \mathrm{n}, \mathrm{bl} / 33 \mathrm{n}$ and $\mathrm{bl} / 32 \mathrm{n}$ ) and in two different laboratories, have very similar composition (Fig. 8).

In the blue samples, cobalt appears linearly well correlated with nickel $\left(R^{2}=0.82\right)$. It seems reasonable to associate these elements to impurities of cobalt pigments. This correlation is in good agreement to the fact that the traditional chromophore used for ceramic pigments with blue tint is the cobalt ion incorporated into different crystalline structures (silicate, alluminate, stannate, cromite, etc.) [29].

It is quite interesting to consider $\mathrm{Co} / \mathrm{As}$ ratio values in order to better understand the technology used for the pigment preparation and to classify the tiles. For the blue decorations, the ratios ranged from 2.0 (sample bl/52a) to 46.1 (sample bl/55a). Low ratios indicate that the samples were not produced in recent years.

Green decorations, except for three samples (v/8a, v/blar, and $\mathrm{vs} / \mathrm{vec})$, contain considerable amounts of copper $(\mathrm{Cu} / \mathrm{Si}=0.055)$ (Fig. 9) suggesting the use of a large number of possible pigments. As one of the strongest coloring elements, $\mathrm{Cu}$ was one of the earliest colorants of enamels in early Egyptian ceramics. It produces a wide range of colors from greenish-blue to green and, in some cases, red depending upon composition and the firing atmosphere of the furnace. Probably green decorations have been obtained using copper oxide that may correspond to the pigment ramina utilized since the Middle Ages.

In all the samples, except in $\mathrm{v} / \mathrm{ve}$, only trace of antimony was detected, instead in the sample $\mathrm{v} / \mathrm{ve}$ the presence of the element $(\mathrm{Sb} / \mathrm{Si}=0.0078)$ could indicate that lead antimonate was used.

Sample v/blar contains elevated quantities of $\mathrm{Sn}(\mathrm{Sn} / \mathrm{Si}=0.27)$, $\mathrm{Mn}(\mathrm{Mn} / \mathrm{Si}=0.025), \mathrm{Fe}(\mathrm{Fe} / \mathrm{Si}=0.022)$ and $\mathrm{Co}(\mathrm{Co} / \mathrm{Si}=0.012)$ and very low copper amounts $(\mathrm{Cu} / \mathrm{Si}=0.0036)$. The green color could be thus a mixture of the yellow (Mars Yellow, Tin yellow, etc.) and blue pigments (Cerulean blue).

The dark green decoration of vs/vec sample contains low amounts of copper $(\mathrm{Cu} / \mathrm{Si}=0.020)$ and elevated amounts of lead $(\mathrm{Pb} / \mathrm{Si}=$ $1.08)$, tin $(\mathrm{Sn} / \mathrm{Si}=0.066)$, manganese $(\mathrm{Mn} / \mathrm{Si}=0.040)$, arsenic $(\mathrm{As} / \mathrm{Si}=0.035)$ and cobalt $(\mathrm{Co} / \mathrm{Si}=0.030)$. Also in this case, to obtain the desired hue, the dark-green color could be produced with a mixture of different pigments. In view of that, the intense green color of the later sample can be ascribed to the intentional addition of $\mathrm{CoO} \cdot \mathrm{nSnO}_{2}+\mathrm{CoSn}(\mathrm{OH})_{6}$ (Blue Cerulean) in combination with $\mathrm{As}_{2} \mathrm{~S}_{3}$ (Orpiment). In this case, considering that the antimony is present only in traces $(\mathrm{Sb} / \mathrm{Si}=0.0079)$, we can exclude the use of Naples Yellow [30,31].

The light green decoration $\mathrm{vc} / \mathrm{vec}$ contains higher amounts of copper $(\mathrm{Cu} / \mathrm{Si}=0.049)$ and iron $(\mathrm{Fe} / \mathrm{Si}=0.062)$ than vs/vec (dark green). In this case, the light green color can be attributed to the addition of a pigment containing copper in combination with an iron base yellow compound. The absence of $\mathrm{Cr}$ in all the green enamels is an indication that no containing chrome pigments (Green of Guignet, green of Lamoriere, etc.) were used in the enamels of the investigated tiles. Also for green color, the relationship Co/As confirms the hypothesized dating by author for nine (v/ve, v/blar, v/1a, v/48a, v/52a, vc/vec, vs/vec, v7/a, v/50a) of the thirteen analyzed green decorations.

Only two red decorations ( $\mathrm{r} / 58 \mathrm{a}, \mathrm{r} / 15 \mathrm{a}$ ) were analyzed. The red sample $\mathrm{r} / 58 \mathrm{a}$ contained large amounts of $\mathrm{Cd},(\mathrm{Cd} / \mathrm{Si}=0.066)$ (Fig. 10) which suggests the use of the pigment CdS. Moreover, the presence of barium $(\mathrm{Ba} / \mathrm{Si}=0.043)$ and manganese $(\mathrm{Mn} / \mathrm{Si}=0.014)$ could suggest the use of low amounts of (psilomelane) $\mathrm{Ba}\left(\mathrm{Mn}^{2+}\right)\left(\mathrm{Mn}^{4+}\right)_{8} \mathrm{O}_{16}(\mathrm{OH})_{4}$ or as $\left(\mathrm{Ba}, \mathrm{H}_{2} \mathrm{O}\right)_{2} \mathrm{Mn}_{5} \mathrm{O}_{10}$ to darken the red decoration (Fig. 1). Unusual is the composition of the red decoration $\mathrm{r} / 15 \mathrm{a}$, where chrome is present to a high level $(\mathrm{Cr} / \mathrm{Si}=0.032)$ which suggests the use of the red chrome pigment $\left(\mathrm{Pb}_{2}(\mathrm{OH})_{2} \cdot \mathrm{CrO}_{4}\right)$. Traces of cobalt were also found $(\mathrm{Co} / \mathrm{Si}=$ 0.0090). An intentional addition of cobalt to obtain a darker hue of the red decoration seems likely.

The pink decoration of sample p57/a contained large amounts of $\mathrm{Pb}$ $(\mathrm{Pb} / \mathrm{Si}=3.7), \mathrm{Sn}(\mathrm{Sn} / \mathrm{Si}=0.41), \mathrm{Mn}(\mathrm{Mn} / \mathrm{Si}=0.33), \mathrm{Ti}(\mathrm{Ti} / \mathrm{Si}=0.29)$ and $\mathrm{Se}(\mathrm{Se} / \mathrm{Si}=0.19)$ (Fig. 10). Moreover, the presence of manganese could suggest the contemporary use of low amounts of a brown pigment to darken the decoration.

From the decoration point of view, when a pastel color is required, as in the case of pink decoration, the ceramist adds an opacifier plus a pigment to the ceramic coating. For example, white lead could have been used in the sample $\mathrm{p} / 57 \mathrm{a}$. In this sample the ratio $\mathrm{Pb} / \mathrm{Si}(\mathrm{Pb} / \mathrm{Si}=3.7)$ is higher than that determined in the other colors (meanly $\mathrm{Pb} / \mathrm{Si}=1.03$ ).

Observation of the data on orange decoration (Fig. 10) reveals a very similar composition to the yellow colored ones. Orange color

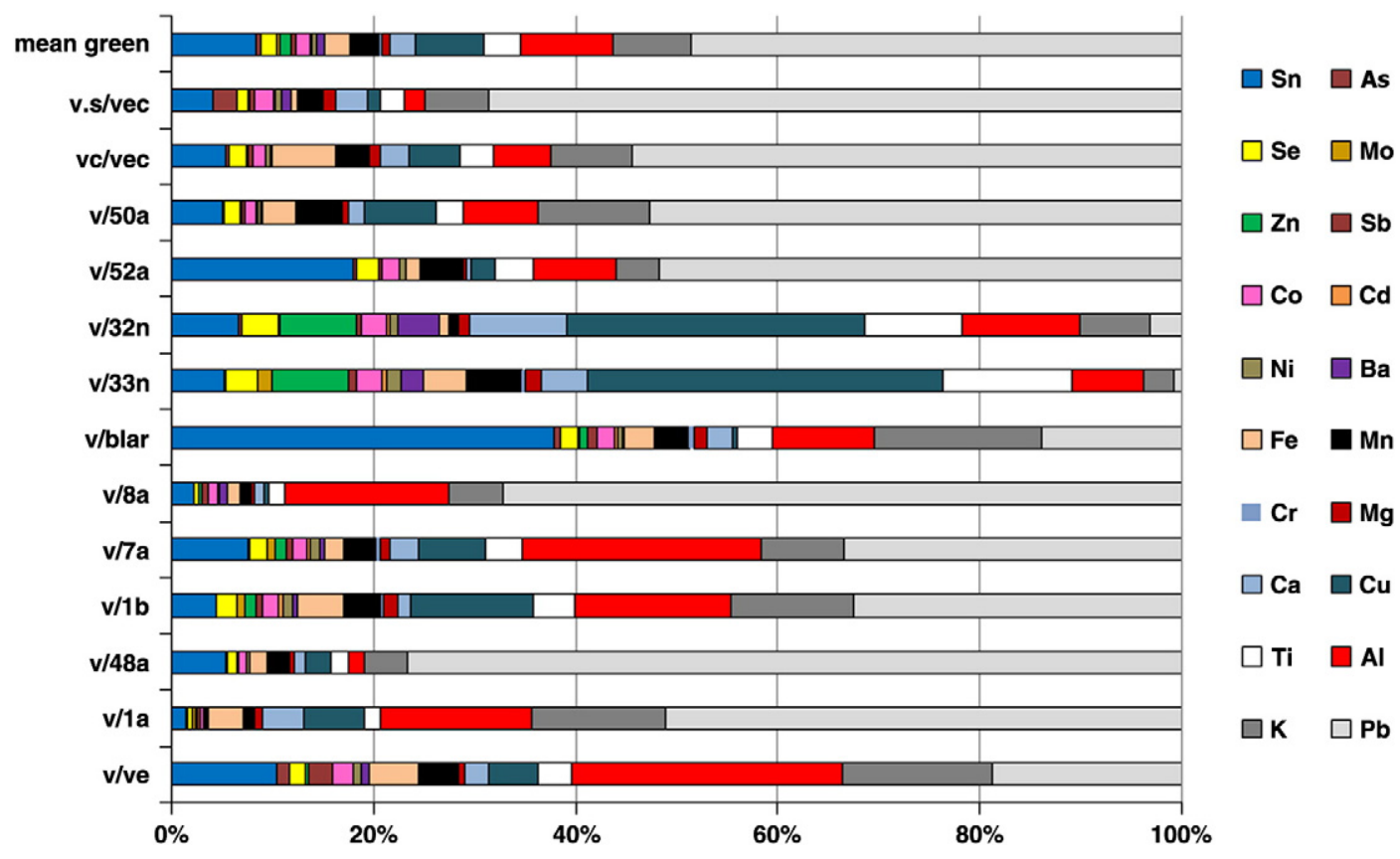

Fig. 9. Chemical composition of the green decorations. 


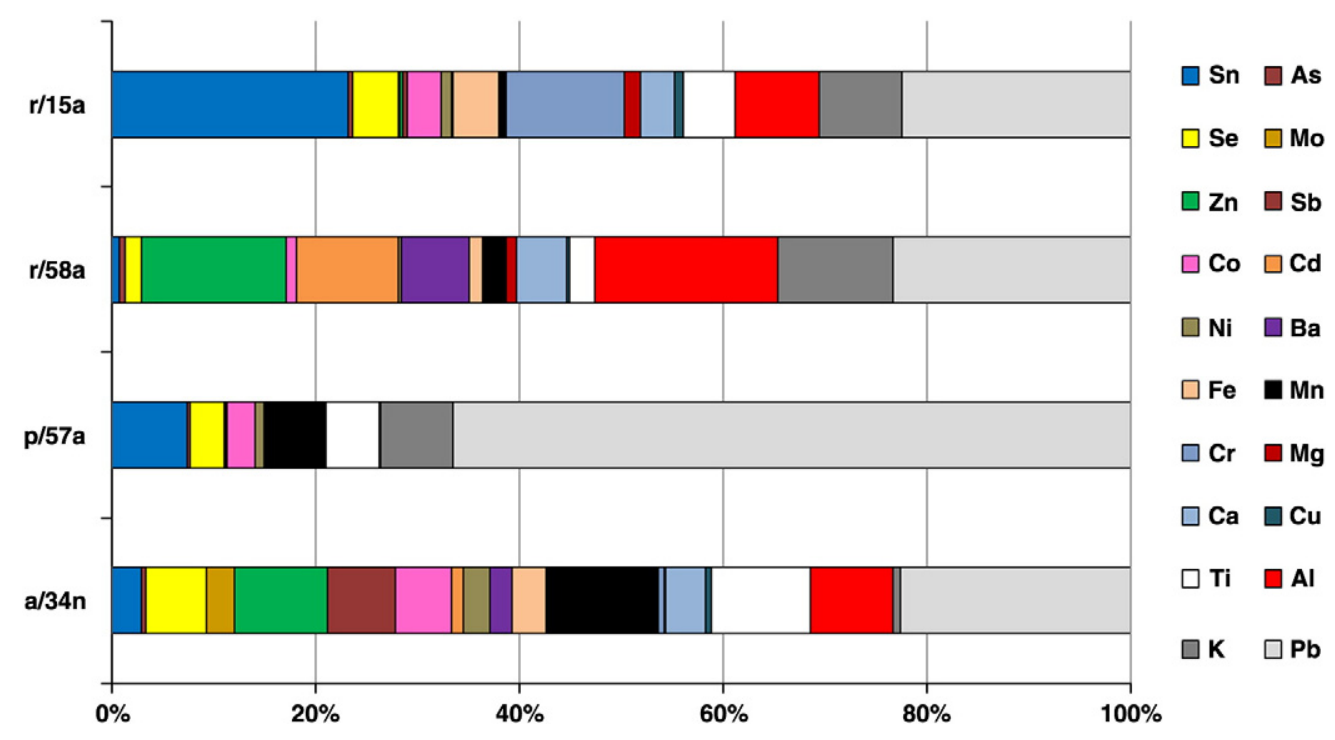

Fig. 10. Chemical composition of the orange $(\alpha / 34 n)$, pink (p/57 $\alpha)$ and red $(r / 58 \alpha, r / 15 \alpha)$ decorations.

present on sample $a / 34 n$, due to the high antimony concentration found $(\mathrm{Sb} / \mathrm{Si}=0.082)$, can be attributed to the use antimony orange $\left(\mathrm{Sb}_{2} \mathrm{O}_{3} \cdot \mathrm{Sb}_{2} \mathrm{~S}\right)$, widely employed for ceramic decoration. As in the case of other pastel decorations, the ceramist added opacifiers to a pigment. For example, $\mathrm{TiO}_{2}(\mathrm{Ti} / \mathrm{Si}=0.12)$ could have been used in the sample $\mathrm{a} / 34 \mathrm{n}$. The presence of titanium confirms the hypothesized dating (21th century) for the sample $34 \mathrm{n}$.

A set of tones between a dark brown and purple is found in the Sicilian tiles. Observation of the brown decorations reveals, except for a sample (m/37a) which has a color similar to purple to brown, a very similar chemical composition (Fig. 11) to the black-colored ones (Fig. 12).

Meanly, brown decorations contain considerable amounts of manganese $(\mathrm{Mn} / \mathrm{Si}=0.076)$ and iron $(\mathrm{Fe} / \mathrm{Si}=0.032)$ which suggests the use of the pigments $\mathrm{MnO}_{2}$ and $\mathrm{Fe}_{2} \mathrm{O}_{3}$. Manganese exists in various oxidation numbers and imparts different derived colors. The tone of these colors in the decorations can be modified by furnace atmosphere. In an oxidant environment the color results pink, but, in a decoration, fired in a reducing atmosphere, deep greenish-brown colors predominate.

It's important to consider that three samples (having the same color) $(\mathrm{m} / 52, \mathrm{~m} / 52 \mathrm{a}, \mathrm{ms} / 52)$, taken from the same tile, show the same composition (Fig. 11). This demonstrates, once again, that the sampling method used by us in this research is reproducible.

Sample $\mathrm{m} / 37$ a differs from the other brown decorations because of its different tone and chemical composition. In this area, the amounts of zinc $(\mathrm{Zn} / \mathrm{Si}=0.085)$, cobalt $(\mathrm{Co} / \mathrm{Si}=0.019)$ and iron $(\mathrm{Fe} / \mathrm{Si}=0.033)$, significantly higher as compared to all other samples, may be due to

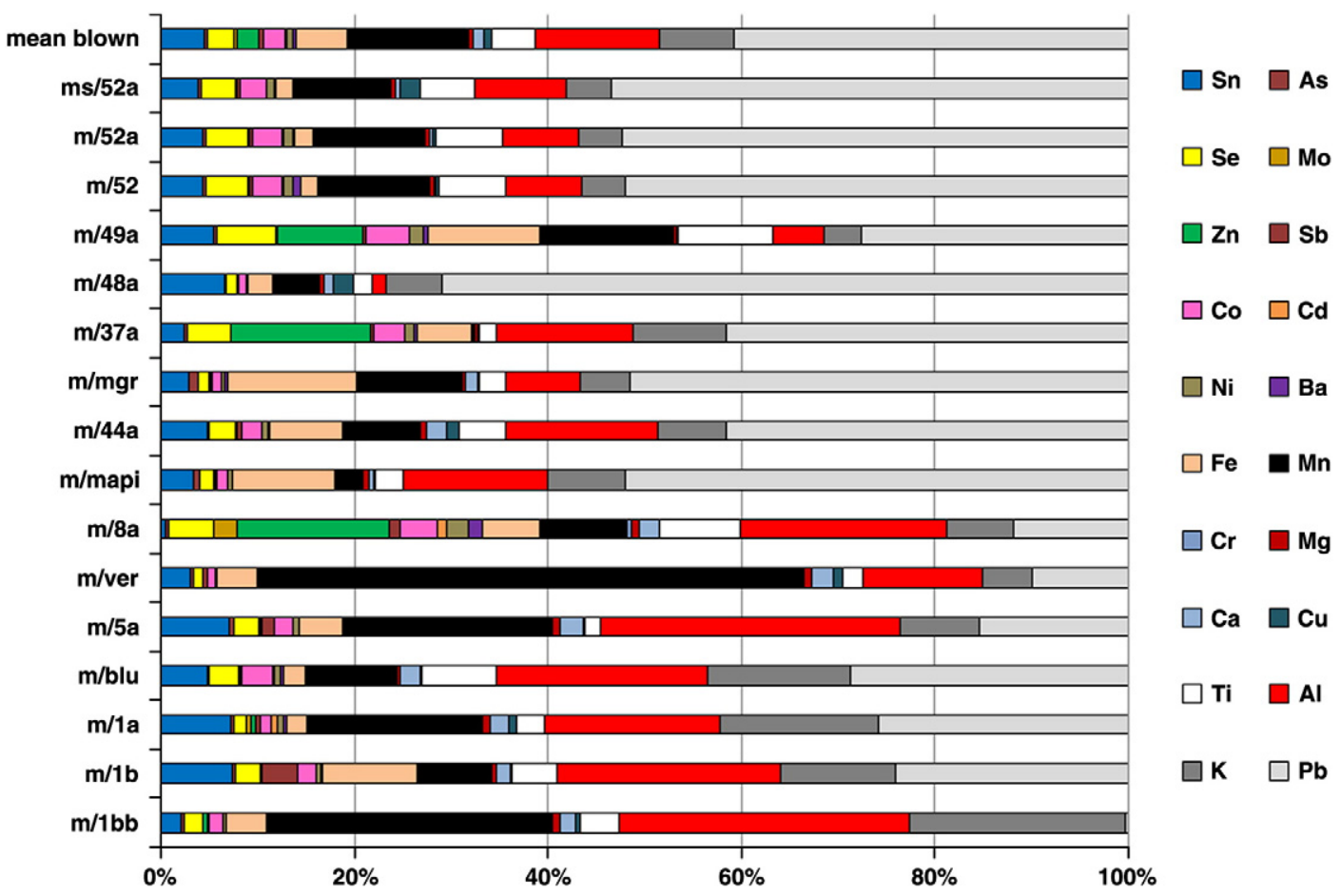

Fig. 11. Chemical composition of the brown decorations. 


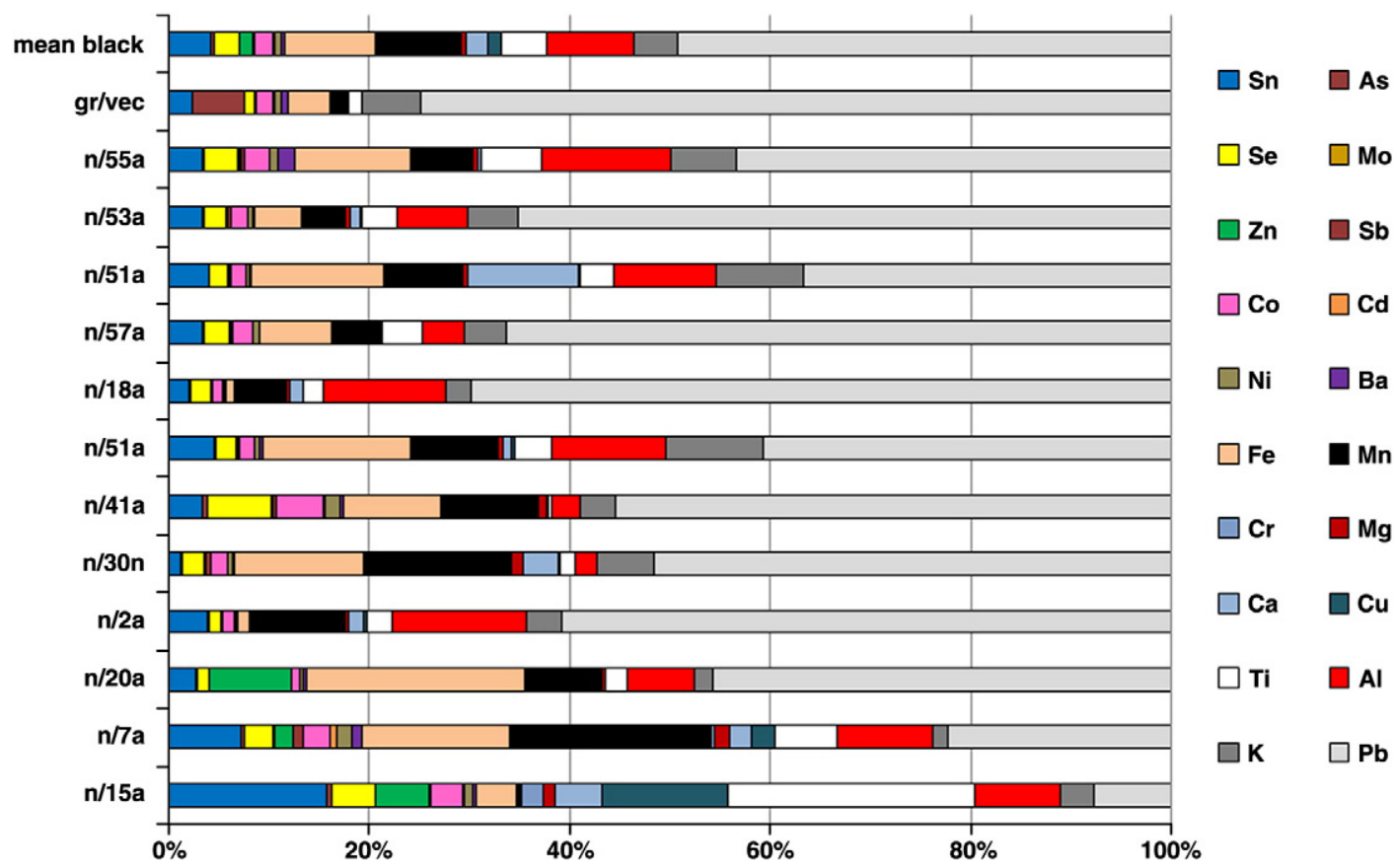

Fig. 12. Chemical composition of the black decorations.

the use of a natural ochre and then deliberately mixed by the ceramist with other pigments, for example zinc oxide and cobalt blue, in order to obtain the desired hues. Differently from all other brown enamels, the sample $\mathrm{m} / 37 \mathrm{a}$ contains manganese only at trace levels $(\mathrm{Mn} / \mathrm{Si}=0.002)$.

The presence of barium $(\mathrm{Ba} / \mathrm{Si}=0.008)$ in sample $\mathrm{m} / 8 \mathrm{a}$ could suggest the use of the mineral psilomelane as a source of manganese, and usually the absence of it implied the use of pyrolusite $\left(\beta-\mathrm{MnO}_{2}\right)[9]$.

Black decorations present in many of the analyzed tiles can be attributed to the use of an iron pigment such as $\mathrm{Fe}_{2} \mathrm{O}_{3}$ (Mars Black) deliberately mixed with $\mathrm{MnO}_{2}$ (Manganese Black), widely employed as pigments for ceramic decoration, in fact the black enamels contain large amounts of iron $(\mathrm{Fe} / \mathrm{Si}=0.068)$ and manganese $(\mathrm{Mn} / \mathrm{Si}=$ 0.062) (Fig. 11).

Gray decorations (gr/vec) contain considerable amounts of arsenic $(\mathrm{As} / \mathrm{Si}=0.055)$, iron $(\mathrm{Fe} / \mathrm{Si}=0.047)$, cobalt $(\mathrm{Co} / \mathrm{Si}=0.020)$ and manganese $(\mathrm{Mn} / \mathrm{Si}=0.020)$. Gray decoration is the most complex recipe, including, probably, four different pigments and several minor components in proportions to obtain the desired hue.

\section{Conclusions}

An analytical approach to the study of ancient tiles is relatively rare. In this study a total of 114 decorations of ceramic floor tiles, manufactured in Sicily in different times from the 16th to the 21th $\mathrm{AD}$, were investigated. Only the ICP-OES technique was used in this study to investigate the elemental composition on the ceramic colored enamels. One of the advantages of the use of ICP-OES for this characterization is the better sensitivity attained, resulting in much improved limits of quantification, which can be particularly relevant for elements that are present at low levels in some of the samples, like chrome, arsenic, and molybdenum.

With the aim to gain some insight onto the practical aspects for sampling decorated tiles, experiments were conducted on different types of decorations (black, light blue and yellow). The micro sampling method, proposed by us, using a cotton swab soaked in hydrofluoric acid, includes advantages of high sensitivity, high selectivity, simplicity, speed, not expensive and can be considered non-destructive because the point of sampling remains invisible to the human eye. The same procedures of sampling and analysis may be used to study enameled ceramics with different shapes and geographical origins (lustro, decorated ceramics from Central Italy, Portuguese Azulejos, Mexican ceramics, etc.).

Enameled decorations in Sicilian tiles were varied in tones and chromatic effects, obtained with a limited number compounds of cobalt, copper, iron, manganese, tin and in the artifacts following the year 1920, also titanium oxide.

An important remark is that some of the colors, such as black, orange, purple and brown were prepared using the same metals, while white, light blue, blue and green were obtained with different elements. Decorations were either composed of pure pigments or prepared as mixtures of pigments following several recipes, to obtain different hues and shades. The majority of the recipes are simple and involve one or two main pigments, sometimes with the addition of opacifiers and other minor components.

The adoption of different recipes for the same color suggests the presence of several laboratories of ceramists working in Sicily who had preferences for different methods for the production of their enameled artifacts.

The aluminum, in the form of oxide or hydroxide, was added to the pigments as a filler, or it is present together with Si, like in many other earth's samples analyzed by ICP-OES [7] as clay.

Concerning the opacifier, the high amounts of lead and antimony, in a case, indicate the use of lead antimonate $\left(\mathrm{Pb}_{2} \mathrm{Sb}_{2} \mathrm{O}_{7}\right)$.

Black decorations were present in several of the analyzed samples and in most of them were identified as being prepared with mixtures of black iron and manganese oxides. The green decorations appear to have been prepared with copper oxide; lighter hue samples contained additional amounts of zinc and/or barium compounds. Yellow decorations are present on many tiles. Considering all the samples, meanly, iron predominates in yellow decorations, only a sample contains large amounts of $\mathrm{Cd}, \mathrm{Zn}$ and very little $\mathrm{Sb}$, which suggests the use of the pigments $\mathrm{ZnO}$ (as white) and $\mathrm{CdS}$ as cadmium yellow. For all samples, except one, we can exclude the use of pigments containing chromium. 


\section{References}

[1] E. Tomasini, G. Siracusano, M.S. Maier, Spectroscopic, morphological and chemical characterization of historic pigments based on carbon. Paths for the identification of an artistic pigment, Microchemical 102 (2012) 28-37.

[2] M. Menu, Analysis of works of art down to the nanometric scale, Microelectron. Eng. 83 (2006) 597-603.

[3] R.J.H. Clark, Pigment identification on Medieval manuscripts by Raman microscopy, J. Mol. Struct. 347 (1995) 417-428.

[4] A. Yoshinari, H. Rodan, K. Tadashi, Y. Ken, N. Akiko, K. Nozomu, Y. Sakuji, N. Izumi, Transition in the use of cobalt-blue colorant in the New Kingdom of Egypt J. Archaeol. Sci. 39 (2012) 1793-1806.

[5] S. Orecchio, Speciation of iron in ancient pots from Sicily (Italy), Microchemical 99 (2011) 132-137.

[6] F. Caruso, S. Orecchio, M.G. Cicero, C. Di Stefano, Gas chromatography-mass spectroscopy characterization of the varnish and glue of an ancient 18th century double bass, J. Chromatogr. A 1147 (2007) 206-212.

[7] M.R. Mannino, S. Orecchio, Chemical characterization of ancient potteries from Himera and Pestavecchia necropolis (Sicily, Italy) by Inductively Coupled Plasma-Optical Emission Spectrometry (ICP-OES), Microchemical 97 (2011) 165-172.

[8] R. Alaimo, G. Bultrini, I. Fragalà, R. Giarrusso, I. Iliopoulos, G. Montana, Archaeometry of Sicilian glazed pottery, Appl. Phys. 79 (2004) 221-227.

[9] S. Coentro, J.M. Mimoso, A.M. Lima, A.S. Silva, A.N. Pais, V.S.F. Muralh, Multi-analytical identification of pigments and pigment mixtures used in 17th century Portuguese Azulejos, J. Eur. Ceram. Soc. 32 (2012) 37-48.

[10] F. Bellanti, M. Tomassetti, G. Visco, L. Campanella, A chemometric approach to the historical and geographical characterization of different terracotta find, Microchemical 88 (2008) 113-120.

[11] D.M. Goltz, J. Coombs, C. Marion, E. Cloutis, J. Gibson, M. Attas, L.-P. Choo-Smith, C. Collins, Pigment identification in artwork using graphite furnace atomic absorption spectrometry, Talanta 63 (2004) 609-616.

[12] P. Fermo, E. Delnevo, M. Lasagni, S.P. Mariette de Vos, Application of chemical and chemometric analytical techniques to the study of ancient ceramics from Dougga (Tunisia), Microchemical 88 (2008) 150-159.

[13] L.J. Holmes, V.J. Robinson, P.R. Makinson, F.R. Livens, Multi-element determination in complex matrices by inductively coupled plasma-mass spectrometry (ICP-MS), Sci. Total Environ. 173 (174) (1995) 345-350.

[14] S. Orecchio, D. Amorello, Platinum levels in urban soils from Palermo (Italy); analytical method using voltammetry, Microchemical 99 (2011) 283-288.

[15] S. Orecchio, D. Amorello, Platinum levels in urban soils from Palermo (Italy); analytical method using voltammetry, Microchemical Journal 99 (2011) 283-288.

[16] R.D. Harley, Artist's Pigments: c. 1600-1835, in: Butterworths Scientific, London, 1970, p. 167.
[17] N.N. Greenwood e, A. Earnshaw, Chemistry of the Elements, Pergamon Press 1985.

[18] D. Gregory Smith, J.H.R. Clark, Raman microscopy in archaeological science, J. Archaeol. Sci. 31 (2004) 1137-1160.

[19] B.H. Berrie, Artists' pigments, in: E.W. Fitzhugh (Ed.), A Handbook of Their History and Characteristics, vol. 3, Oxford University Press, New York, 1997, pp. 191-339.

[20] J. Dik, E. Hermens, R. Peschar, H. Schenk, Early production recipes for lead antimonate yellow in Italian art, Archaeometry 47 (2005) 593-607.

[21] F. Rosi, V. Manuali, C. Miliani, B.G. Brunetti, A. Sgamellotti, T. Grygar, Raman scattering features of lead pyroantimonate compounds. Part I: XRD and Raman characterization of Pb2Sb2O7 doped with tin and zinc, J. Raman Spectrosc. 40 (2009) 107-111

[22] F. Rosi, V. Manuali, T. Grygar, P. Bezdicka, B.G. Brunetti, A. Sgamellotti, Raman scattering features of lead pyroantimonate compounds: implication for the non-invasive identification of yellow pigments on ancient ceramics. Part II. In situ characterization of Renaissance plates by portable micro-Raman and XRF studies, J. Raman Spectrosc. 42 (2011) 407-414.

[23] R.L. Feller (Ed.), Artists' pigments, A Handbook of Their History and Characteristics, vol. 1, National Gallery of Art, Washington DC, 1986.

[24] B. Karasu, S. Turan, Effects of cobalt, copper, manganese and titanium oxide addition on the microstructures of zinc containing soft porcelain glazes, Eur. Ceram. Soc. 22 (2002) 1447-1455.

[25] B. Gratuze, I. Soulier, J.N. Barrandon, D. Foy, De l'origine du cobalt dans les verres, Revue d'Archéométrie 16 (1992) 97-108.

[26] B. Gratuze, M. Picon, Utilisation par l'industrie verrière des sels d'aluns des oasis égyptiennes au début du premier millénaire avant notre ère, in: J.-P. Brun (Ed.), L'Alun de Mediterranie, Institut Français de Naples, 2006, pp. 269-276.

[27] O. Schalm, V. Van der Linden, P. Frederickx, S. Luyten, G. Van der Snickt, J. Caen, D. Schryvers, K. Janssens, E. Cornelis, D. Van Dyck, M. Schreiner, Enamels in stained glass windows: preparation, chemical composition, microstructure and causes of deterioration, Spectrochim. Acta B 64 (2009) 812-820.

[28] Dictionary of Arts, Manufactures, and Mines: Containing a Clear Exposition of Their Principles, Andrew Ure, Published 1844, D. Appleton \& Co.

[29] Classification and Chemical Description of the Complex Inorganic Colour Pigments, in: Dry Color Manufacturer's Association, Alexandria, VA, 1991, p. 23.

[30] K. Sakellariou, C. Miliani, A. Morresi, M. Ombelli, Spectroscopic investigation of yellow majolica glazes, J. Raman Spectrosc. 35 (2004) 61-67.

[31] J. Miao, B. Yang, D. Mu, Identification and differentiation of opaque Chinese over glaze yellow enamels by Raman spectroscopy and supporting techniques, Archaeometry 52 (2010) 146-155. 